\title{
Observable set, observability, interpolation inequality and spectral inequality for the heat equation in $\mathbb{R}^{n}$
}

\author{
Gengsheng Wang* Ming Wang ${ }^{\dagger}$ Can Zhang ${ }^{\ddagger}$ Yubiao Zhang ${ }^{\S}$
}

November 12, 2017

\begin{abstract}
This paper studies connections among observable sets, the observability inequality, the Hölder-type interpolation inequality and the spectral inequality for the heat equation in $\mathbb{R}^{n}$. We present a characteristic of observable sets for the heat equation. In more detail, we show that a measurable set in $\mathbb{R}^{n}$ satisfies the observability inequality if and only if it is $\gamma$-thick at scale $L$ for some $\gamma>0$ and $L>0$. We also build up the equivalence among the above-mentioned three inequalities. More precisely, we obtain that if a measurable set $E \subset \mathbb{R}^{n}$ satisfies one of these inequalities, then it satisfies others. Finally, we get some weak observability inequalities and weak interpolation inequalities where observations are made over a ball.
\end{abstract}

Keywords: Characteristic of observable sets, observability inequality, Hölder-type interpolation inequality, spectral inequality, heat equation

AMS subject classifications: 49J20, 49K20, 93D20

\section{Contents}

1 Introduction $\quad 2$

1.1 Thick sets and several inequalities . . . . . . . . . . . . . . . . . . . . . 2

1.2 Aim, motivation and main result . . . . . . . . . . . . . . . . . . . . . . . . . . . . .

1.3 Extensions to bounded observable sets . . . . . . . . . . . . . . . . . . . 8

1.4 Plan of the paper . . . . . . . . . . . . . . . . . . . 9

2 Proof of Theorem 1.1 $r$

3 Weak interpolation and observability inequalities $\quad 22$

3.1 Weak interpolation inequalities with observation on the unit ball . . . . . . . . . 22

3.2 Weak observability inequalities with observations on balls . . . . . . . . . . . . 35

*School of Mathematics and Statistics, Wuhan University, Wuhan, 430072, China. Email: wanggs62@yeah.net

${ }^{\dagger}$ School of Mathematics and Physics, China University of Geosciences, Wuhan, 430074, China. Email: mwangcug@outlook.com

${ }^{\ddagger}$ School of Mathematics and Statistics, Wuhan University, Wuhan, 430072, China, and Department of Mathematics, University of the Basque Country (UPV/EHU), Bilbao, 48080, Spain. Email: zhangcansx@163.com

$\S$ Center for Applied Mathematics, Tianjin University, Tianjin, 300072, China. Email: yubiao-zhang@yeah.net 


\section{Introduction}

In this paper, we consider the heat equation in the whole physical space $\mathbb{R}^{n}$ :

$$
\partial_{t} u-\triangle u=0 \quad \text { in }(0, \infty) \times \mathbb{R}^{n}, \quad u(0, \cdot) \in L^{2}\left(\mathbb{R}^{n}\right) .
$$

For this equation, we will characterize the observable sets and build up connections among several important inequalities which are introduced in the next subsection.

Notation Write $C(\cdots)$ for a positive constant that depends on what are included in the brackets and may vary in different contexts. The same can be said about $C^{\prime}(\cdots), C_{1}(\cdots)$ and so on. Use $V_{n}$ to denote the volume of the unit ball in $\mathbb{R}^{n}$. Let $B_{r}(x)$, with $x \in \mathbb{R}^{n}$ and $r>0$, be the ball in $\mathbb{R}^{n}$, centered at $x$ and of radius $r$. (Simply write $B_{r}=B_{r}(0)$.) Let $\mathbb{S}^{n-1}$ be the unit spherical surface in $\mathbb{R}^{n}$. Denote by $Q$ the open unit cube in $\mathbb{R}^{n}$, centered at the origin. Let $x+L Q$, with $x \in \mathbb{R}^{n}$ and $L>0$, be the set $\{x+L y: y \in Q\}$. For each measurable $D \subset \mathbb{R}^{n}$, denote by $|D|$ its Lebesgue measure. Given $f \in L^{2}\left(\mathbb{R}^{n}\right)$, write $\widehat{f}$ for its Fourier transform ${ }^{1}$. Let $\left\{e^{t \Delta}: t \geq 0\right\}$ be the semigroup generated by the Laplacian operator in $\mathbb{R}^{n}$. Given $x=\left(x_{1}, \ldots, x_{n}\right) \in \mathbb{R}^{n}$, let $|x|:=\left(\sum_{i=1}^{n} x_{i}^{2}\right)^{1 / 2}$ and $\langle x\rangle:=\sqrt{1+|x|^{2}}$.

\subsection{Thick sets and several inequalities}

We start with introducing sets of $\gamma$-thick at scale $L$.

Sets of $\gamma$-thick at scale $L \quad A$ measurable set $E \subset \mathbb{R}^{n}$ is said to be $\gamma$-thick at scale $L$ for some $\gamma>0$ and $L>0$, if

$$
|E \bigcap(x+L Q)| \geq \gamma L^{n} \text { for each } x \in \mathbb{R}^{n} .
$$

About sets of $\gamma$-thick at scale $L$, several remarks are given in order.

$\left(a_{1}\right)$ To our best knowledge, this definition arose from studies of the uncertainty principle. We quote it from [5] (see Page 5 in [5]). Before [5], some very similar concepts were proposed. For instance, the definition of relative dense sets was given in [23] (see also Page 113 in [19]); the definition of thick sets was introduced in [24].

$\left(a_{2}\right)$ Each set $E$ of $\gamma$-thick at scale $L$ has the following properties: First, in each cube with the length $L,|E|$ is bigger than or equals to $\gamma L^{n}$. Consequently, $E$ has a positive Lebesgue measure in each neighborhood of every $x$ in $\mathbb{R}^{n}$. Second, $E$ is also a set of $\gamma$-thick at scale $2 L$, but the reverse is not true. Third, we necessary have that $\gamma \leq 1$.

Next, we introduce an observability inequality for the equation (1.1).

The observability inequality $A$ measurable set $E \subset \mathbb{R}^{n}$ is said to satisfy the observability inequality for the equation (1.1), if for any $T>0$ there exists a positive constant $C_{o b s}=C_{o b s}(n, T, E)$ so that when $u$ solves (1.1),

$$
\int_{\mathbb{R}^{n}}|u(T, x)|^{2} \mathrm{~d} x \leq C_{o b s} \int_{0}^{T} \int_{E}|u(t, x)|^{2} \mathrm{~d} x \mathrm{~d} t .
$$

\footnotetext{
${ }^{1}$ Given $f$ in the Schwartz class $\mathcal{S}\left(\mathbb{R}^{n}\right)$, its Fourier transform is as: $\widehat{f}(\xi)=(2 \pi)^{-n} \int_{\mathbb{R}^{n}} e^{-i x \xi} f(x) \mathrm{d} x, \xi \in \mathbb{R}^{n}$. Since $\mathcal{S}\left(\mathbb{R}^{n}\right)$ is dense in $L^{2}\left(\mathbb{R}^{n}\right)$, by a standard way, we can define $\widehat{f}$ for each $f \in L^{2}\left(\mathbb{R}^{n}\right)$.
} 
When a measurable $E \subset \mathbb{R}^{n}$ satisfies (1.3), it is called an observable set for (1.1).

Several notes on the observability inequality (1.3) are given in order.

$\left(b_{1}\right)$ Being treated the integral on the left hand side as a recovering term, and the integral on the right hand side as an observation term, the inequality (1.3) can be understood as: one can recover a solution of (1.1) at time $T$, via observing it on the set $E$ and in the time interval $(0, T)$. From perspective of control theory, the inequality (1.3) is equivalent to the following null controllability: For any $u_{0} \in L^{2}\left(\mathbb{R}^{n}\right)$ and $T>0$, there exists a control $f \in L^{2}\left((0, T) \times \mathbb{R}^{n}\right)$ driving the solution $u$ to the controlled equation: $\partial_{t} u-\triangle u=\chi_{E} f$ in $(0, T) \times \mathbb{R}^{n}$, from the initial state $u_{0}$ to the state 0 at time $T$. (Here, $\chi_{E}$ is the characteristic function of $E$.)

$\left(b_{2}\right)$ We can compare (1.3) with the observability inequality for the heat equation on a bounded physical domain. Let $\Omega$ be a bounded $C^{2}$ (or Lipschitz and locally star-shaped, see [2]) domain in $\mathbb{R}^{n}$. Consider the equation:

$$
\begin{cases}\partial_{t} u-\triangle u=0 & \text { in }(0, \infty) \times \Omega \\ u=0 & \text { on }(0, \infty) \times \partial \Omega \\ u(0, \cdot) \in L^{2}(\Omega) . & \end{cases}
$$

We say a measurable set $\omega \subset \Omega$ satisfies the observability inequality for (1.4), if given $T>0$, there is a constant $C(n, T, \omega, \Omega)>0$ so that when $u$ solves (1.4),

$$
\int_{\Omega}|u(T, x)|^{2} \mathrm{~d} x \leq C(n, T, \omega, \Omega) \int_{0}^{T} \int_{\omega}|u(t, x)|^{2} \mathrm{~d} x \mathrm{~d} t .
$$

When a measurable set $\omega \subset \Omega$ satisfies (1.5), it is called an observable set for (1.4).

The inequality (1.5) has been widely studied. See $[18,27,32]$ for the case where $\omega$ is open; $[1,2,16]$ for the case when $\omega$ is measurable.

$\left(b_{3}\right)$ When $\Omega$ is an unbounded domain and $\omega$ is bounded and open subset of $\Omega$, the inequality (1.5) may not be true. This was showed in [35] for the heat equation in the physical domain $\mathbb{R}^{+}$. Similar results have been obtained for higher dimension cases in [36]. For the heat equation in an unbounded domain, [37] imposed a condition, in terms of the Gaussian kernel, on the set $\omega$ so that the observability inequality does not hold. In particular, [37] showed that the observability inequality fails when $|\omega|<\infty$. Notice that for the heat equation (1.1), any set $E$ of finite measure does not have the characteristic on observable sets of (1.1). This characteristic is indeed the $\gamma$-thickness at scale $L$ for some $\gamma>0$ and $L>0$. (See Theorem 1.1 of this paper.)

About works on sufficient conditions of observable sets for heat equations in unbounded domains, we would like to mention the work [7]. It showed that, for some parabolic equations in an unbounded domain $\Omega \subset \mathbb{R}^{n}$, the observability inequality holds when the observation is made over a subset $E \subset \Omega$, with $\Omega \backslash E$ bounded. For other similar results, we refer the reader to [38]. When $\Omega=\mathbb{R}^{n}$, such a set $E$ has the characteristic on observable sets of (1.1) mentioned before.

$\left(b_{4}\right)$ An interesting phenomenon is that some potentials (growing at infinity) in heat equations may change the above-mentioned characteristic on observable sets for the heat equations with potentials. In $[39,9]$, the authors realized the following fact: Let $A=\triangle+V$, where $V(x):=-|x|^{2 k}, x \in \mathbb{R}^{n}$, with $2 \leq k \in \mathbb{N}$. Write $\left\{e^{t A}\right\}_{t \geq 0}$ for the semigroup generated by 
the operator $A$. Let $r_{0} \geq 0$ and let $\Theta_{0}$ be an open subset of $\mathbb{S}^{n-1}$. Let $\Gamma=\left\{x \in \mathbb{R}^{n}:|x| \geq\right.$ $\left.r_{0}, x /|x| \in \Theta_{0}\right\}$. Then there is $C\left(n, T, \Theta_{0}, r_{0}, k\right)>0$ so that

$$
\int_{\mathbb{R}^{n}}\left|e^{T A} u_{0}\right|^{2} \mathrm{~d} x \leq C\left(n, T, \Theta_{0}, r_{0}, k\right) \int_{0}^{T} \int_{\Gamma}\left|e^{t A} u_{0}\right|^{2} \mathrm{~d} x \mathrm{~d} t \text { for all } u_{0} \in L^{2}\left(\mathbb{R}^{n}\right) .
$$

The cone $\Gamma$ does not have the characteristic on observable sets mentioned before, but still holds the observability inequality (1.6). The main reason is that the unbounded potential $V$ plays an important role in the proof of $(1.6)$ (see $[39,9]$ ). It should be pointed out that when $V(x)=-|x|, x \in \mathbb{R}^{n}$ (which means that the potential grows more slowly at infinity), (1.6) does not hold for the above cone. We refer the readers to [39, 9] for more details on this issue. Besides, we also would like to mention [3] for this subject.

An interesting question now arises: How do potentials influence characteristics of observable sets? We wish to answer this question in our future studies.

We then introduce an interpolation inequality for the equation (1.1).

The Hölder-type interpolation inequality $A$ measurable set $E \subset \mathbb{R}^{n}$ is said to satisfy the Hölder-type interpolation inequality for the heat equation (1.1), if for any $\theta \in(0,1)$, there is $C_{H o l d}=C_{H o l d}(n, E, \theta)>0$ so that for each $T>0$ and each solution $u$ to the equation (1.1),

$$
\int_{\mathbb{R}^{n}}|u(T, x)|^{2} \mathrm{~d} x \leq e^{C_{\text {Hold }}\left(1+\frac{1}{T}\right)}\left(\int_{E}|u(T, x)|^{2} \mathrm{~d} x\right)^{\theta}\left(\int_{\mathbb{R}^{n}}|u(0, x)|^{2} \mathrm{~d} x\right)^{1-\theta} .
$$

Several remarks on the Hölder-type interpolation inequality (1.7) are given in order.

$\left(c_{1}\right)$ The inequality (1.7) is a kind of quantitative unique continuation for the heat equation (1.1). It provides a Hölder-type propagation of smallness for solutions of the heat equation (1.1). In fact, if $\int_{E}|u(T, x)|^{2} \mathrm{~d} x=\delta$, then we derive from (1.7) that $\int_{\mathbb{R}^{n}}|u(T, x)|^{2} \mathrm{~d} x$ is bounded by $C \delta^{\theta}$ for some constant $C>0$. Letting $\delta \rightarrow 0$, we find that $u(T, x)=0$ for almost every $x \in \mathbb{R}^{n}$.

$\left(c_{2}\right)$ From perspective of control theory, the inequality (1.7) implies the approximate null controllability with cost for impulse controlled heat equations, i.e., for any $T>\tau>0$, $\varepsilon>0$ and any $u_{0} \in L^{2}\left(\mathbb{R}^{n}\right)$, there is $f \in L^{2}\left(\mathbb{R}^{n}\right)$ and $C=C(n, E, T, \tau, \varepsilon)>0$ so that

$$
\|f\|_{L^{2}\left(\mathbb{R}^{n}\right)} \leq C\left\|u_{0}\right\|_{L^{2}\left(\mathbb{R}^{n}\right)} \text { and }\|u(T, \cdot)\|_{L^{2}\left(\mathbb{R}^{n}\right)} \leq \varepsilon\left\|u_{0}\right\|_{L^{2}\left(\mathbb{R}^{n}\right)}
$$

where $u$ is the solution to the impulse controlled equation: $\partial_{t} u-\Delta u=\delta_{\{t=\tau\}} \chi_{E} f$ in $(0, T) \times$ $\mathbb{R}^{n}$, with the initial condition $u(0, x)=u_{0}(x), x \in \mathbb{R}^{n}$. (See [45, Theorem 3.1].)

$\left(c_{3}\right)$ The Hölder-type interpolation inequality (1.7) can imply the observability inequality (1.3). Moreover, it leads to the following stronger version of (1.3):

$$
\int_{\mathbb{R}^{n}}|u(T, x)|^{2} \mathrm{~d} x \leq C_{o b s} \int_{F} \int_{E}|u(t, x)|^{2} \mathrm{~d} x \mathrm{~d} t, \quad \text { with } C_{o b s}=C_{o b s}(n, T, E, F),
$$

where $F \subset(0, T)$ is a subset of positive measure. This will be presented in Lemma 2.4. Our methods used to prove (1.8) are very similar to those developed in [44, 46, 2] where heat equations on bounded domains are considered. 
$\left(c_{4}\right)$ We can compare (1.7) with an interpolation inequality for heat equation (1.4). A measurable set $\omega \subset \Omega$ is said to satisfy the Hölder-type interpolation inequality for the equation (1.4), if for any $\theta \in(0,1)$ and $T>0$, there is $C=C(n, T, \Omega, \omega, \theta)$ so that when $u$ solves (1.4),

$$
\int_{\Omega}|u(T, x)|^{2} \mathrm{~d} x \leq C\left(\int_{\omega}|u(T, x)|^{2} \mathrm{~d} x\right)^{\theta}\left(\int_{\Omega}|u(0, x)|^{2} \mathrm{~d} x\right)^{1-\theta} .
$$

In [43], the authors proved that any open and nonempty subset $\omega \subset \Omega$ satisfies the Höldertype interpolation inequality (1.9) for heat equations with potentials in bounded and convex domains. The frequency function method used in [43] were partially borrowed from [11]. In [2], the authors proved that any subset $\omega$ of positive measure satisfies the Hölder-type interpolation inequality (1.9) for the heat equation (1.4) where $\Omega$ is a bounded Lipschitz and locally star-shaped domain in $\mathbb{R}^{n}$. More about this inequality for heat equations in bounded domains, we referee the readers to $[44,45,46]$.

Finally, we will introduce a spectral inequality for some functions in $L^{2}\left(\mathbb{R}^{n}\right)$.

The spectral inequality $A$ measurable set $E \subset \mathbb{R}^{n}$ is said to satisfy the spectral inequality, if there is a positive constant $C_{\text {spec }}=C_{\text {spec }}(n, E)$ so that for each $N>0$,

$$
\int_{\mathbb{R}^{n}}|f(x)|^{2} \mathrm{~d} x \leq e^{C_{\text {spec }}(1+N)} \int_{E}|f(x)|^{2} \mathrm{~d} x \text { for all } f \in L^{2}\left(\mathbb{R}^{n}\right) \text { with supp } \widehat{f} \subset B_{N} .
$$

Several notes on the spectral inequality (1.10) are given in order.

$\left(d_{1}\right)$ Recall the Lebeau-Robbiano spectral inequality (see [27, 28]): Let $\Omega$ be a bounded smooth domain in $\mathbb{R}^{n}$ and let $\omega$ be a nonempty open subset of $\Omega$. Write $\triangle_{\Omega}$ for the Laplacian operator on $L^{2}(\Omega)$ with Domain $\left(\triangle_{\Omega}\right)=H_{0}^{1}(\Omega) \bigcap H^{2}(\Omega)$. Let $\left\{\lambda_{j}\right\}_{j \geq 1}$ (with $\left.\lambda_{1}<\lambda_{2} \leq \cdots\right)$ be the eigenvalues of $-\triangle_{\Omega}$ and let $\left\{\phi_{j}\right\}_{j \geq 1}$ be the corresponding eigenfunctions. Then there is a positive constant $C(\Omega, \omega)$ so that

$$
\int_{\Omega}|f(x)|^{2} \mathrm{~d} x \leq e^{C(\Omega, \omega)(1+\sqrt{\lambda})} \int_{\omega}|f(x)|^{2} \mathrm{~d} x \text { for all } f \in \operatorname{span}\left\{\phi_{j} ; \lambda_{j} \leq \lambda\right\} .
$$

This inequality was extended to the case where $\Omega$ is a bounded $C^{2}$ domain via a simpler way in [33]. Then it was extended to the case that $\Omega$ is a bounded Lipschitz and locally star-shaped domain, $\omega$ is a subset of positive measure and $C(\Omega, \omega)=C(\Omega,|\omega|$ ) (see Theorem 5 in [2]).

By our understanding, the inequality (1.10) is comparable to (1.11) from two perspectives as follows: First, the inequality (1.10) is made for the subspace:

$$
E_{N} \triangleq\left\{g \in L^{2}\left(\mathbb{R}^{n}\right): \operatorname{supp} \widehat{g} \subset B_{N}\right\} \text { with } N>0,
$$

while the inequality (1.11) is made for the subspace:

$$
F_{\lambda} \triangleq\left\{\sum_{\lambda_{j} \leq \lambda} a_{j} e_{j} \in L^{2}(\Omega):\left\{a_{j}\right\}_{j \geq 1} \subset \mathbb{R}\right\} \quad \text { with } \lambda>0 .
$$

From the definition of the spectral projection in the abstract setting given in [48] (see Pages 262-263 in [48]), we can define two spectral projections: $\chi_{\left[0, N^{2}\right]}(-\Delta)$ and $\chi_{[0, \lambda]}\left(-\Delta_{\Omega}\right)$ on $L^{2}\left(\mathbb{R}^{n}\right)$ and $L^{2}(\Omega)$, respectively. Then after some computations, we find that $E_{N}$ and $F_{\lambda}$ are the ranges of $\chi_{\left[0, N^{2}\right]}(-\Delta)$ and $\chi_{[0, \lambda]}\left(-\Delta_{\Omega}\right)$, respectively. Second, the square root of the integral of $\chi_{\left[0, N^{2}\right]}$ over $\mathbb{R}$ is $N$ which corresponds to the $N$ in (1.10), while the square root of the integral of $\chi_{[0, \lambda]}$ over $\mathbb{R}$ is $\sqrt{\lambda}$ which corresponds to the $\sqrt{\lambda}$ in (1.11). 
$\left(d_{2}\right)$ Though the inequality (1.10) was first named as the spectral inequality in [30] (to our best knowledge), it has been studied for long time. (See, for instance, [5, 19, 23, 24, 40, 31, 41, 42, 49].) In [24], the author announced that if $E$ is $\gamma$-thick at scale $L$ for some $\gamma>0$ and $L>0$, then $E$ satisfies the spectral inequality (1.10), and further proved this announcement for the case when $n=1$. Earlier, the authors of [31] proved that $E$ is $\gamma$-thick at scale $L$ for some $\gamma>0$ and $L>0$ if and only if $E$ satisfies the following inequality: For each $N>0$, there is a positive constant $C(n, E, N)$ so that

$$
\int_{\mathbb{R}^{n}}|f(x)|^{2} \mathrm{~d} x \leq C(n, E, N) \int_{E}|f(x)|^{2} \mathrm{~d} x \text { for each } f \in L^{2}\left(\mathbb{R}^{n}\right) \text { with supp } \widehat{f} \subset B_{N} .
$$

This result is often referred as the Logvinenko-Sereda theorem. Before [31], the above equivalence was proved by B. P. Paneyah for the case that $n=1$ (see $[42,41,19]$ ).

The constant $C(n, E, N)$ in (1.12) was not expressed explicitly in terms of $N$ in [31]. From this point of view, (1.12) is weaker than the spectral inequality (1.10). In the proof of our main theorem of this paper, the expression $C(n, E, N)=e^{C_{\text {spec }}(1+N)}$ will play an important role.

$\left(d_{3}\right)$ The inequality (1.12) is also important. It is closely related to the uncertainty principle (which is an extensive research topic in the theory of harmonic analysis and says roughly that a nonzero function and its Fourier transform cannot be both sharply localized, see [17]). In fact, a set $E$ of $\gamma$-thick at scale $L$ satisfies the inequality (1.12) if and only if it satisfies the following uncertainty principle:

$$
\int_{\mathbb{R}^{n}}|f(x)|^{2} \mathrm{~d} x \leq C^{\prime}(n, E, N)\left(\int_{E}|f(x)|^{2} \mathrm{~d} x+\int_{B_{N}^{c}}|\widehat{f}(\xi)|^{2} \mathrm{~d} \xi\right) \text { for all } f \in L^{2}\left(\mathbb{R}^{n}\right),
$$

where $C^{\prime}(n, E, N)>0$ and $B_{N}^{c}$ is the complement set of $B_{N}$ in $\mathbb{R}^{n}$. We refer the interested readers to $[5,19,40]$ for the proof of the above result, as well as more general uncertainty principle, where $E$ and $B_{N}^{c}$ are replaced by more general sets.

It deserves mentioning what follows: The uncertainty principle can help us to get the exact controllability for the Schrödinger equation with controls located outside of two balls and at two time points. This was realized in [52]. (See [25] for more general cases.)

$\left(d_{4}\right)$ By using a global Carleman estimate, the authors in [30] proved the spectral inequality (1.10) for such an open subset $E$ that satisfies the property: there exists $\delta>0$ and $r>0$ so that

$$
\forall y \in \mathbb{R}^{n}, \exists y^{\prime} \in E \text { such that } B_{r}\left(y^{\prime}\right) \subset E \text { and }\left|y-y^{\prime}\right| \leq \delta .
$$

It is clear that a set with the above property (1.13) is a set of $\gamma$-thick at scale $L$ for some $\gamma>0$ and $L>0^{2}$.

\subsection{Aim, motivation and main result}

Aim According to the note $\left(d_{2}\right)$ in the previous subsection, the characteristic of a measurable set holding the spectral inequality (1.10) is the $\gamma$-thickness at scale $L$ for some $\gamma>0$ and $L>0$. Natural and interesting questions are as follows: What is the characteristic of observable sets for (1.1)? How to characterize a measurable set $E$ satisfying the Hölder-type interpolation inequality (1.7)? What are the connections among inequalities (1.3), (1.7) and (1.10)? The aim of this paper is to answer the above questions.

Motivation The motivations of our studies are given in order.

\footnotetext{
${ }^{2}$ In fact, one can choose $L=2(\delta+r), \gamma=r^{n}(2(\delta+r))^{-n} V_{n}$.
} 
(i) The first motivation arises from two papers [4] and [1]. In [4], the authors gave, for the wave equation in a bounded physical domain $\Omega \subset \mathbb{R}^{n}$, a sufficient and almost necessary condition to ensure an open subset $\Gamma \subset \partial \Omega$ to be observable, (i.e., $\Gamma$ satisfies the observability inequality for the wave equation with observations on $\Gamma$ ). This condition is exactly the well known Geometric Control Condition (GCC for short) ${ }^{3}$. Thus, we can say that the GCC condition is a characteristic of observable open sets on $\partial \Omega$, though this condition is not strictly necessary (see [29]). The authors in [1] presented a sufficient and necessary condition to ensure a measurable subset $\omega \subset \Omega$ satisfying (1.5). This condition is as: $|\omega|>0$. Hence, the characteristic of observable sets for the equation (1.4) is as: $|\omega|>0$.

Analogically, it should be very important to characterize observable sets for the heat equation (1.1). However, it seems for us that there is no any such result in the past publications. These motivate us to find the characteristic of observable sets for the equation (1.1).

(ii) For the heat equation (1.4), the observability inequality (1.5), the Hölder-type interpolation inequality (1.9) and the spectral inequality (1.11) are equivalent. More precisely, we have that if $\omega \subset \Omega$ is a measurable set, then

$$
|\omega|>0 \Longleftrightarrow \omega \text { satisfies }(1.11) \Longleftrightarrow \omega \text { satisfies }(1.9) \Longleftrightarrow \omega \text { satisfies }(1.5) \text {. }
$$

The proof of (1.14) was hidden in the paper [2]. (See Theorem 5, Theorem 6, as well as its proof, Theorem 1, as well as its proof, in [2].) However, for the heat equation (1.1), the equivalence among these three inequalities has not been touched upon. These motivate us to build up the equivalence among inequalities (1.3), (1.7) and (1.10).

It deserves mentioning that for heat equations with lower terms in bounded physical domains, we do not know if (1.14) is still true.

Main Result The main result of the paper is the next Theorem 1.1.

Theorem 1.1. Let $E \subset \mathbb{R}^{n}$ be a measurable subset. Then the following statements are equivalent.

(i) The set $E$ is $\gamma$-thick at scale $L$ for some $\gamma>0$ and $L>0$.

(ii) The set E satisfies the spectral inequality (1.10).

(iii) The set E satisfies the Hölder-type interpolation inequality (1.7).

(iv) The set E satisfies the observability inequality (1.3).

Several remarks about Theorem 1.1 are given in order.

$\left(e_{1}\right)$ The equivalence of statements (i) and (iv) in Theorem 1.1 tells us: the characteristic of observable sets for the heat equation (1.1) is the $\gamma$-thickness at scale $L$ for some $\gamma>0$ and $L>0$. This seems to be new for us.

$\left(e_{2}\right)$ The equivalence among statements (ii), (iii) and (iv) in Theorem 1.1 presents closed connections of the three inequalities. This seems also to be new for us.

$\left(e_{3}\right)$ We find the following way to prove Theorem 1.1: (i) $\Rightarrow(\mathbf{i i}) \Rightarrow(\mathbf{i i i}) \Rightarrow(\mathbf{i v}) \Rightarrow(\mathbf{i})$. We prove $(\mathbf{i}) \Rightarrow$ (ii) by some ideas from [24]. Indeed, this result was announced in [24] and then proved for the case that $n=1$ in the same reference. We prove (ii) $\Rightarrow$ (iii) $\Rightarrow$ (iv), though using some ideas and techniques from $[2,44]$. Finally, we show (iv) $\Rightarrow(\mathbf{i})$ via the structure of a special solution to the equation(1.1).

\footnotetext{
${ }^{3}$ Recall that an open subset $\omega \subset \Omega$ is said to satisfy the GCC if there exists $T_{0}$ such that any geodesic with velocity one meets $\omega$ within time $T_{0}$ (see e.g. [26]).
} 


\subsection{Extensions to bounded observable sets}

From Theorem 1.1, we see that in order to have (1.3) or (1.7), the set $E$ has to be $\gamma$-thick at scale $L$ for some $\gamma>0$ and $L>0$. Then a natural and interesting question arises: What are possible substitutions of (1.3) or (1.7), when $E$ is replaced by a ball in $\mathbb{R}^{n}$ ? (It deserves to mention that any ball in $\mathbb{R}^{n}$ does not satisfy the thick condition (1.2).) We try to find the substitutes from two perspectives as follows:

(i) We try to add weights on the left hand side and ask ourself if the following inequalities hold for all solutions of (1.1):

$$
\int_{\mathbb{R}^{n}} \chi_{B_{r^{\prime}}}(x)|u(T, x)|^{2} \mathrm{~d} x \leq C\left(T, r^{\prime}, r, n\right) \int_{0}^{T} \int_{B_{r}}|u(t, x)|^{2} \mathrm{~d} x \mathrm{~d} t
$$

and

$$
\int_{\mathbb{R}^{n}} \rho(x)|u(T, x)|^{2} \mathrm{~d} x \leq C(T, \rho, r, n) \int_{0}^{T} \int_{B_{r}}|u(t, x)|^{2} \mathrm{~d} x \mathrm{~d} t
$$

where $\rho(x)=\langle x\rangle^{-\nu}$ or $e^{-|x|}$. On one hand, we proved that (1.15) is true when $r^{\prime}<r$, while (1.15) is not true when $r^{\prime}>r$ (see Theorem 3.2 in Subsection 3.2). Unfortunately, we do not know if (1.15) holds when $r^{\prime}=r$. On the other hand, we showed that (1.16) fails for all $r>0$ (see Corollary 3.2 in Subsection 3.2).

(ii) We try to find a class of initial data so that (1.3) (where $E$ is replaced by $B_{r}$ ) holds for all solutions of (1.1) with initial data in this class. We have obtained some results on this issue (see Theorem 3.3 in Subsection 3.2). More interesting question is as: what is the biggest class of initial data so that (1.3) (where $E$ is replaced by $B_{r}$ ) holds for all solutions of the heat equation (1.4) with initial data in this class? Unfortunately, we are not able to answer it.

We now turn to possible substitutions of (1.7) where $E$ is replaced by $B_{1}$. We expect that for each $\varepsilon \in(0,1)$, there are $C(n, T)>0$ and $b(\varepsilon)>0$ so that when $u$ solves (1.1),

$$
\int_{\mathbb{R}^{n}}|u(T, x)|^{2} \mathrm{~d} x \leq C(n, T)\left(\varepsilon \int_{\mathbb{R}^{n}}|u(0, x)|^{2} \mathrm{~d} x+b(\varepsilon) \int_{B_{1}}|u(T, x)|^{2} \mathrm{~d} x\right) .
$$

Let us explain why (1.17) deserves to be expected. Reason One. Let $\theta \in(0,1)$ and $T>0$. Then the next two inequalities are equivalent. The first inequality is as: there is $C(n, T, \theta)$ so that when $u$ solves (1.1),

$$
\int_{\mathbb{R}^{n}}|u(T, x)|^{2} \mathrm{~d} x \leq C(n, T, \theta)\left(\int_{B_{1}}|u(T, x)|^{2} \mathrm{~d} x\right)^{\theta}\left(\int_{\mathbb{R}^{n}}|u(0, x)|^{2} \mathrm{~d} x\right)^{1-\theta},
$$

while the second inequality is as: there is $C(n, T, \theta)>0$ so that for any $\varepsilon \in(0,1)$ and any solution $u$ to $(1.1)$,

$$
\int_{\mathbb{R}^{n}}|u(T, x)|^{2} \mathrm{~d} x \leq C(n, T, \theta)\left(\varepsilon \int_{\mathbb{R}^{n}}|u(0, x)|^{2} \mathrm{~d} x+\varepsilon^{-\frac{1-\theta}{\theta}} \int_{E}|u(T, x)|^{2} \mathrm{~d} x\right) .
$$

However, (1.19) is not true, for otherwise, we can use the same method developed in [44] (see also [2]) to derive (1.3) (where $E$ is replaced by $B_{1}$ ) which contradicts the equivalence of (i) and (iv) in Theorem 1.1. Thus, $b(\varepsilon)$ in (1.17) cannot grow like a polynomial of $\varepsilon$. But it seems that we still have such a hope that (1.17) holds for some kind of $b(\varepsilon)$. Reason Two. 
The space-like strong unique continuation of the heat equation (1.1) (see [11]) yields that if $u(T, \cdot)=0$ on the ball $B_{1}$, then $u(T, \cdot)=0$ over $\mathbb{R}^{n}$. The inequality (1.17) is a quantitative version of the aforementioned unique continuation.

Though we have not found any $b(\varepsilon)$ so that $(1.17)$ is true, we obtained some $b(\varepsilon)$ so that (1.17) holds for all solutions to (1.1) with initial data having some slight decay (see Theorem 3.1 in Subsection 3.1).

Finally, We would like to mention what follows: With the aid of an abstract lemma (i.e., Lemma 5.1) in [52], each of extended inequalities mentioned above corresponds to a kind of controllability for the heat equation (1.1). We are not going to repeat the details on this issue in the current paper.

\subsection{Plan of the paper}

The paper is organized as follows: In Section 2, we prove Theorem 1.1. In Section 3, we present several weak observability inequalities and weak interpolation inequalities, where observations are made in a ball of $\mathbb{R}^{n}$.

\section{Proof of Theorem 1.1}

We are going to prove Theorem 1.1 in the following way:

$$
(\mathbf{i}) \Rightarrow(\text { ii }) \Rightarrow(\text { iii }) \Rightarrow(\text { iv }) \Rightarrow(\mathbf{i}) \text {. }
$$

The above steps are based on several lemmas: Lemmas 2.1, 2.3, 2.4 and 2.5. We begin with Lemma 2.1 connecting the spectral inequality with sets of $\gamma$-thick at scale $L$.

Lemma 2.1. Suppose that a measurable set $E \subset \mathbb{R}^{n}$ is $\gamma$-thick at scale $L$ for some $\gamma>0$ and $L>0$. Then E satisfies the spectral inequality (1.10), with

$$
C_{\text {spec }}(n, E)=C(1+L)\left(1+\ln \frac{1}{\gamma}\right) \text { for some } C=C(n) .
$$

Remark 2.1. The manner that the constant $e^{C_{s p e c}(n, E)(1+N)}$ (in (1.10)) depends on $N$ is comparable with how the constant $e^{C \sqrt{\lambda}}$ in (1.11) depends on $\lambda$. The latter plays an important role in the proof of the Hölder-type interpolation inequality (1.9) for the heat equation (1.4) (see [2]).

Remark 2.2. In [24], the author announced the result in Lemma 2.1 and proved it for the case when $n=1$. For the completeness of the paper, we give a detailed proof for Lemma 2.1, based on some ideas and techniques in [24].

To show Lemma 2.1, we need the following result on analytic functions:

Lemma 2.2 ([24, Lemma 1]). Let $\Phi(z)$ be an analytic function in $D_{5}(0)$ (the disc in $\mathbb{C}$, centered at origin and of radius 5). Let $I$ be an interval of length 1 such that $0 \in I$. Let $\hat{E} \subset I$ be a subset of positive measure. If $|\Phi(0)| \geq 1$ and $M=\max _{|z| \leq 4}|\Phi(z)|$, then there exists a generic constant $C>0$ such that

$$
\sup _{x \in I}|\Phi(x)| \leq(C /|\hat{E}|)^{\frac{\ln M}{\ln 2}} \sup _{x \in \hat{E}}|\Phi(x)| .
$$

We now on the position to prove Lemma 2.1. 
Proof of Lemma 2.1. We only need to prove this lemma for the case when $L=1$. In fact, suppose that this is done. Let $E$ be $\gamma$-thick at scale $L>0$. Define a new set:

$$
L^{-1} E:=\left\{L^{-1} x: x \in E\right\} .
$$

One can readily check that $L^{-1} E$ is $\gamma$-thick at scale 1 . Given $N>0$ and $f \in L^{2}\left(\mathbb{R}^{n}\right)$ with $\operatorname{supp} \widehat{f} \subset B_{N}$, let

$$
g(x):=f(L x), x \in \mathbb{R}^{n} .
$$

One can directly check that

$$
\widehat{g}(\xi)=L^{-n} \widehat{f}\left(L^{-1} \xi\right), \xi \in \mathbb{R}^{n} ; \quad \operatorname{supp} \widehat{g} \subset B_{L N} .
$$

From these, we can apply Lemma 2.1 (with $L=1$ ) to the set $L^{-1} E$ and the function $g$ to find $C=C(n)$ so that

$$
\begin{aligned}
\int_{\mathbb{R}^{n}}|g(x)|^{2} \mathrm{~d} x & \leq e^{2 C\left(1+\ln \frac{1}{\gamma}\right)(1+L N)} \int_{L^{-1} E}|g(x)|^{2} \mathrm{~d} x \\
& \leq e^{2 C(1+L)\left(1+\ln \frac{1}{\gamma}\right)(1+N)} \int_{L^{-1} E}|g(x)|^{2} \mathrm{~d} x .
\end{aligned}
$$

Meanwhile, by changing variable $x \mapsto L x$, we deduce that

$$
\int_{\mathbb{R}^{n}}|f(x)|^{2} \mathrm{~d} x=L^{-n} \int_{\mathbb{R}^{n}}|g(x)|^{2} \mathrm{~d} x ; \quad \int_{E}|f(x)|^{2} \mathrm{~d} x=L^{-n} \int_{L^{-1} E}|g(x)|^{2} \mathrm{~d} x .
$$

Hence, from (2.1) and (2.2), we find that

$$
\int_{\mathbb{R}^{n}}|f(x)|^{2} \mathrm{~d} x \leq e^{C_{s p e c}(n, E)(1+N)} \int_{E}|f(x)|^{2} \mathrm{~d} x,
$$

with

$$
C_{\text {spec }}(n, E)=2 C(1+L)\left(1+\ln \frac{1}{\gamma}\right) .
$$

This proves the lemma for the general case that $L>0$.

We now show Lemma 2.1 for the case when $L=1$ by several steps. First of all, we arbitrarily fix $N>0$ and $f \in L^{2}\left(\mathbb{R}^{n}\right)$ with supp $\widehat{f} \subset B_{N}$. Without loss of generality, we can assume that $f \neq 0$.

Step 1. Bad and good cubes. For each multi-index $j=\left(j_{1}, j_{2}, \cdots, j_{n}\right) \in \mathbb{Z}^{n}$, let

$$
Q(j):=\left\{x=\left(x_{1}, \ldots, x_{n}\right) \in \mathbb{R}^{n}:\left|x_{i}-j_{i}\right|<1 / 2 \text { for all } i=1,2, \cdots, n\right\} .
$$

It is clear that

$$
Q(j) \bigcap Q(k)=\varnothing \text { for all } j \neq k \in \mathbb{Z}^{n} ; \mathbb{R}^{n}=\bigcup_{j \in \mathbb{Z}^{n}} \overline{Q(j)},
$$

where $\overline{Q(j)}$ denotes the closure of $Q(j)$. From these, we have that

$$
\int_{\mathbb{R}^{n}}|f(x)|^{2} \mathrm{~d} x=\sum_{j \in \mathbb{Z}^{n}} \int_{Q(j)}|f(x)|^{2} \mathrm{~d} x .
$$


We will divide $\left\{Q(j): j \in \mathbb{Z}^{n}\right\}$ into two disjoint parts whose elements are respectively called "good cubes" and "bad cubes". And then we compare $\int_{\mathbb{R}^{n}}|f|^{2}$ with $\int_{\cup Q(j) \text { is bad }}|f|^{2}$ and $\int_{\cup Q(j) \text { is good }}|f|^{2}$, respectively. First, we define the function:

$$
h(s):=s^{n}(s-1)^{-n}-1, s \in[2,+\infty) .
$$

It is a continuous and strictly decreasing function satisfying that

$$
h(2) \geq 1, \quad \lim _{s \rightarrow+\infty} h(s)=0 .
$$

Thus we can take $A_{0}$ as the unique point in $[2,+\infty)$ so that $h\left(A_{0}\right)=1 / 2$. Clearly, $A_{0}$ depends only on $n$, i.e., $A_{0}=A_{0}(n)$. Given $j \in \mathbb{Z}^{n}, Q(j)$ is said to be a good cube, if for each $\beta \in \mathbb{N}^{n}$,

$$
\int_{Q(j)}\left|\partial_{x}^{\beta} f(x)\right|^{2} \mathrm{~d} x \leq A_{0}^{|\beta|} N^{2|\beta|} \int_{Q(j)}|f(x)|^{2} \mathrm{~d} x .
$$

When $Q(j)$ is not a good cube, it is said to be a bad cube. Thus, when $Q(j)$ is a bad cube, there is $\beta \in \mathbb{N}^{n}$, with $|\beta|>0$, so that

$$
\int_{Q(j)}\left|\partial_{x}^{\beta} f(x)\right|^{2} \mathrm{~d} x>A_{0}^{|\beta|} N^{2|\beta|} \int_{Q(j)}|f(x)|^{2} \mathrm{~d} x .
$$

Using the Plancherel theorem and the assumption that supp $\widehat{f} \subset B_{N}(0)$, we obtain that for each $\beta \in \mathbb{N}^{n}$,

$$
\begin{aligned}
\int_{\mathbb{R}^{n}}\left|\partial_{x}^{\beta} f(x)\right|^{2} \mathrm{~d} x & =\int_{\mathbb{R}^{n}}\left|\widehat{\partial_{x}^{\beta} f(x)}\right|^{2} \mathrm{~d} \xi=\int_{\mathbb{R}^{n}}\left|(i \xi)^{\beta} \widehat{f}(\xi)\right|^{2} \mathrm{~d} \xi=\int_{|\xi| \leq N}\left|\xi^{\beta} \widehat{f}(\xi)\right|^{2} \mathrm{~d} \xi \\
& \leq N^{2|\beta|} \int_{|\xi| \leq N}|\widehat{f}(\xi)|^{2} \mathrm{~d} \xi=N^{2|\beta|} \int_{\mathbb{R}^{n}}|f(x)|^{2} .
\end{aligned}
$$

Meanwhile, it follows by (2.5) that when $Q(j)$ is a bad cube,

$$
\int_{Q(j)}|f(x)|^{2} \mathrm{~d} x \leq \sum_{\beta \in \mathbb{N}^{n},|\beta|>0} A_{0}^{-|\beta|} N^{-2|\beta|} \int_{Q(j)}\left|\partial_{x}^{\beta} f(x)\right|^{2} \mathrm{~d} x .
$$

Since $Q(j), j \in \mathbb{Z}^{n}$, are disjoint, by taking the sum in (2.7) for all bad cubes, we find that

$$
\begin{aligned}
\int_{\cup Q(j) \text { is bad }}|f(x)|^{2} \mathrm{~d} x & \leq \sum_{\beta \in \mathbb{N}^{n},|\beta|>0} A_{0}^{-|\beta|} N^{-2|\beta|} \int_{\bigcup Q(j) \text { is bad }}\left|\partial_{x}^{\beta} f(x)\right|^{2} \mathrm{~d} x \\
& \leq \sum_{\beta \in \mathbb{N}^{n},|\beta|>0} A_{0}^{-|\beta|} N^{-2|\beta|} \int_{\mathbb{R}^{n}}\left|\partial_{x}^{\beta} f(x)\right|^{2} \mathrm{~d} x .
\end{aligned}
$$

From (2.6) and (2.8), we have that

$$
\begin{aligned}
\int_{\bigcup Q(j) \text { is bad }}|f(x)|^{2} \mathrm{~d} x & \leq \sum_{\beta \in \mathbb{N}^{n},|\beta|>0} A_{0}^{-|\beta|} \int_{\mathbb{R}^{n}}|f(x)|^{2} \mathrm{~d} x \\
& =\left(A_{0}^{n}\left(A_{0}-1\right)^{-n}-1\right) \int_{\mathbb{R}^{n}}|f(x)|^{2} \mathrm{~d} x .
\end{aligned}
$$


Since $h\left(A_{0}\right)=\frac{1}{2}$, it follows from (2.9) that

$$
\int_{\cup Q(j) \text { is bad }}|f(x)|^{2} \mathrm{~d} x \leq \frac{1}{2} \int_{\mathbb{R}^{n}}|f(x)|^{2} \mathrm{~d} x .
$$

By (2.3) and (2.10), we obtain that

$$
\int_{\bigcup Q(j) \text { is good }}|f(x)|^{2} \mathrm{~d} x \geq \frac{1}{2} \int_{\mathbb{R}^{n}}|f(x)|^{2} \mathrm{~d} x .
$$

Step 2. Properties on good cubes. Arbitrarily fix a good cube $Q(j)$. We will prove some properties related to $Q(j)$. First of all, we claim that there is $C_{0}(n)>0$ so that

$$
\left\|\partial_{x}^{\beta} f\right\|_{L^{\infty}(Q(j))} \leq C_{0}(n)(1+N)^{n}\left(\sqrt{A_{0}} N\right)^{|\beta|}\|f\|_{L^{2}(Q(j))} \text { for all } \beta \in \mathbb{N}^{n} .
$$

In fact, according to $(2.4)$, there is $C_{1}(n)>0$ so that

$$
\begin{aligned}
\left\|\partial_{x}^{\beta} f\right\|_{W^{n, 2}(Q(j))} & =\sum_{|\mu| \leq n}\left(\int_{Q(j)}\left|\partial_{x}^{\beta+\mu} f(x)\right|^{2} \mathrm{~d} x\right)^{1 / 2} \\
& \leq \sum_{|\mu| \leq n} A_{0}^{\frac{|\beta+\mu|}{2}} N^{|\beta+\mu|}\|f\|_{L^{2}(Q(j))} \\
& \leq C_{1}(n)(1+N)^{n}\left(\sqrt{A_{0}} N\right)^{|\beta|}\|f\|_{L^{2}(Q(j))} \text { for all } \beta \in \mathbb{N}^{n} . .
\end{aligned}
$$

Meanwhile, because $Q(j)$ satisfies the cone condition, we can apply the Sobolev embedding theorem $W^{n, 2}(Q(j)) \hookrightarrow L^{\infty}(Q(j))$ to get that for some $C_{2}(n)>0$,

$$
\|\varphi\|_{L^{\infty}(Q(j))} \leq C_{2}(n)\|\varphi\|_{W^{n, 2}(Q(j))} \text { for all } \varphi \in W^{n, 2}(Q(j)) .
$$

This, along with (2.13), leads to (2.12).

Next, we let $y \in \overline{Q(j)}$ satisfy that

$$
\|f\|_{L^{\infty}(Q(j))}=|f(y)| .
$$

(Due to the continuity of $|f|$ over $\mathbb{R}^{n}$, such $y$ exists.) Because the diameter of $Q(j)$ is $\sqrt{n}$, we can use the spherical coordinates centered at $y$ to obtain that

$$
\begin{aligned}
|E \bigcap Q(j)| & =\int_{0}^{\infty} d r \int_{|x-y|=r} \chi_{E \cap Q(j)}(x) d \sigma \\
& =\int_{0}^{\sqrt{n}} d r \int_{|x-y|=r} \chi_{E \cap Q(j)}(x) d \sigma \\
& =\sqrt{n} \int_{0}^{1} d r \int_{|x-y|=\sqrt{n} r} \chi_{E \cap Q(j)}(x) d \sigma .
\end{aligned}
$$

In (2.15), we change the variable:

$$
x=y+\sqrt{n} r w \text { with } w \in \mathbb{S}^{n-1},
$$

and then obtain that

$$
|E \bigcap Q(j)|=\sqrt{n} \int_{0}^{1}(\sqrt{n} r)^{n-1} d r \int_{\mathbb{S}^{n-1}} \chi_{E \bigcap Q(j)}(y+\sqrt{n} r w) d \sigma
$$




$$
\leq n^{n / 2} \int_{0}^{1} d r \int_{\mathbb{S}^{n-1}} \chi_{E \cap Q(j)}(y+\sqrt{n} r w) d \sigma
$$

For each $w \in \mathbb{S}^{n-1}$, let

$$
I_{w} \triangleq\{r \in[0,1]: y+\sqrt{n} r w \in E \bigcap Q(j)\} .
$$

Since

$$
\left|\mathbb{S}^{n-1}\right|=\frac{2 \pi^{\frac{n}{2}}}{\Gamma\left(\frac{n}{2}\right)}, \text { where } \Gamma(\cdot) \text { is the Gamma function, }
$$

it follows from (1.2) and (2.16) that

$$
\left|I_{\omega_{0}}\right| \geq \frac{|E \bigcap Q(j)|}{n^{n / 2}\left|\mathbb{S}^{n-1}\right|} \geq \frac{\Gamma\left(\frac{n}{2}\right)}{2(n \pi)^{n / 2}} \gamma \text { for some } w_{0} \in \mathbb{S}^{n-1} .
$$

Then we define a function $\phi(\cdot)$ over $[0,1]$ by

$$
\phi(t)=\frac{f\left(y+\sqrt{n} t w_{0}\right)}{\|f\|_{L^{2}(Q(j))}}, t \in[0,1] .
$$

(Since $f \in L^{2}\left(\mathbb{R}^{n}\right)$ satisfies that supp $\widehat{f} \subset B_{N}$, we have that $f$ is analytic over $\mathbb{R}^{n}$. Consequently, $\|f\|_{L^{2}(Q(j))} \neq 0$ because we assumed that $f \neq 0$ over $\mathbb{R}^{n}$.)

We claim that $\phi(t)$ can be extended to be an entire function in the complex plane. In fact, by (2.19), one can directly check that

$$
\left|\phi^{(k)}(0)\right| \leq \frac{n^{\frac{3}{2} k} \max _{|\beta|=k}\left\|D^{\beta} f\right\|_{L^{\infty}(Q(j))}}{\|f\|_{L^{2}(Q(j))}} \text { for all } k \geq 0 .
$$

By (2.20) and (2.12), we see that

$$
\left|\phi^{(k)}(0)\right| \leq C_{0}(n)(1+N)^{n}\left(n^{\frac{3}{2}} \sqrt{A_{0}} N\right)^{k} \text { for all } k \geq 0 .
$$

From (2.21), we find that

$$
\phi(t)=\phi(0)+\phi^{\prime}(0) t+\cdots+\frac{\phi^{(k)}(0)}{k !} t^{k}+\cdots, \quad t \in[0,1],
$$

and that the series in (2.22), with $t$ being replaced by any $z \in \mathbb{C}$, is convergent. Thus, the above claim is true. From now on, we will use $\phi(z)$ to denote the extension of $\phi(t)$ over $\mathbb{C}$.

Step 3. Recovery of the $L^{2}\left(\mathbb{R}^{n}\right)$ norm. We will finish our proof in this step. Applying Lemma 2.2 , where

$$
I=[0,1], \quad \hat{E}=I_{w_{0}}(\text { defined by (2.17) and (2.18)) and } \Phi=\phi,
$$

and then using (2.18), we can find $C_{3}=C_{3}(n)$ so that

$$
\begin{aligned}
\sup _{t \in[0,1]}|\phi(t)| & \leq\left(C /\left|I_{w_{0}}\right|\right)^{\frac{\ln M}{\ln 2}} \sup _{t \in I_{w_{0}}}|\phi(t)| \\
& \leq\left(\frac{2 C(n \pi)^{n / 2}}{\gamma \Gamma\left(\frac{n}{2}\right)}\right)^{\frac{\ln M}{\ln 2}} \sup _{t \in I_{w_{0}}}|\phi(t)| \\
& \leq M^{C_{3}\left(1+\ln \frac{1}{\gamma}\right)} \sup _{t \in I_{w_{0}}}|\phi(t)|,
\end{aligned}
$$


where

$$
M=\max _{|z| \leq 4}|\phi(z)| .
$$

Two facts are given in order. First, it follows from (2.14) and (2.19) that

$$
\frac{\|f\|_{L^{\infty}(Q(j))}}{\|f\|_{L^{2}(Q(j))}}=\frac{|f(y)|}{\|f\|_{L^{2}(Q(j))}}=|\phi(0)| .
$$

Second, it follows by the definition of $I_{w_{0}}$ that

$$
\sup _{t \in I_{w_{0}}}|\phi(t)| \leq \frac{\|f\|_{L^{\infty}(E \bigcap Q(j))}}{\|f\|_{L^{2}(Q(j))}} .
$$

The above two facts, along with (2.23), yield that

$$
\|f\|_{L^{\infty}(Q(j))} \leq M^{C_{3}\left(1+\ln \frac{1}{\gamma}\right)}\|f\|_{L^{\infty}(E \bigcap Q(j))} .
$$

We next define

$$
E^{\prime} \triangleq\left\{x \in E \bigcap Q(j):|f(x)| \leq \frac{2}{|E \bigcap Q(j)|} \int_{E \bigcap Q(j)}|f(x)| \mathrm{d} x\right\} .
$$

By the Chebyshev inequality, we have that

$$
\left|E^{\prime}\right| \geq \frac{|E \bigcap Q(j)|}{2} \geq \frac{\gamma}{2} .
$$

By the same argument as that used in the proof of (2.25), one can obtain that

$$
\|f\|_{L^{\infty}(Q(j))} \leq M^{C_{4}\left(1+\ln \frac{1}{\gamma}\right)}\|f\|_{L^{\infty}\left(E^{\prime} \cap Q(j)\right)} \text { for some } C_{4}=C_{4}(n) .
$$

Meanwhile, it follows by the definition of $E^{\prime}$ that

$$
\|f\|_{L^{\infty}\left(E^{\prime} \cap Q(j)\right)} \leq \frac{2}{|E \bigcap Q(j)|} \int_{E \cap Q(j)}|f(x)| \mathrm{d} x .
$$

From (2.26), (2.27) and the Hölder inequality, we find that

$$
\int_{Q(j)}|f|^{2} \mathrm{~d} x \leq \frac{4}{\gamma} M^{2 C_{4}\left(1+\ln \frac{1}{\gamma}\right)} \int_{E \cap Q(j)}|f|^{2} \mathrm{~d} x .
$$

The term $M$ (given by (2.24)) can be estimated by (2.21) as follows:

$$
\begin{aligned}
M & =\max _{|z| \leq 4}|\phi(z)| \\
& \leq \max _{|z| \leq 4} \sum_{k=0}^{\infty} \frac{\phi^{(k)}(0)}{k !}|z|^{k} \\
& \leq C_{2}(n)(1+N)^{n} \sum_{k=0}^{\infty} \frac{\left(4 n^{\frac{3}{2}} \sqrt{A_{0}} N\right)^{k}}{k !} \\
& \leq e^{C_{5}(1+N)} \text { for some } C_{5}=C_{5}(n) .
\end{aligned}
$$


Finally, combining (2.28) and (2.29) leads to that

$$
\int_{Q(j)}|f|^{2} \mathrm{~d} x \leq e^{C_{6}(1+N)\left(1+\ln \frac{1}{\gamma}\right)} \int_{E \bigcap Q(j)}|f|^{2} \mathrm{~d} x \text { for some } C_{6}=C_{6}(n) .
$$

Taking the sum in (2.30) for all good cubes, using (2.11), we see that

$$
\begin{aligned}
\int_{\mathbb{R}^{n}}|f|^{2} \mathrm{~d} x & \leq 2 \sum_{Q(j) \text { is good }} \int_{Q(j)}|f|^{2} \mathrm{~d} x \\
& \leq 2 \sum_{Q(j) \text { is good }} e^{C_{6}(1+N)\left(1+\ln \frac{1}{\gamma}\right)} \int_{E \cap Q(j)}|f|^{2} \mathrm{~d} x \\
& \leq 2 e^{C_{6}(1+N)\left(1+\ln \frac{1}{\gamma}\right)} \int_{E}|f|^{2} \mathrm{~d} x
\end{aligned}
$$

which leads to (1.10), with

$$
C_{\text {spec }}(n, E)=C_{6}(1+L)\left(1+\ln \frac{1}{\gamma}\right) .
$$

This ends the proof of Lemma 2.1.

Lemma 2.3 and Lemma 2.4 deal with connections among the spectral inequality (1.10), the Hölder-type interpolation inequality (1.7) and the observability inequality (1.8). In their proofs, we borrowed some ideas and techniques from [2, 44].

Lemma 2.3. Suppose that a measurable set $E \subset \mathbb{R}^{n}$ satisfies the spectral inequality (1.10). Then E satisfies the Hölder-type interpolation (1.7), with

$$
C_{\text {Hold }}=\frac{1}{1-\theta}\left(C_{\text {spec }}+1\right)^{2}+\ln 12 .
$$

Proof. Let $E \subset \mathbb{R}^{n}$ satisfy the spectral inequality (1.10). Arbitrarily fix $T>0, \theta \in(0,1)$ and a solution $u$ to (1.1). Write $u(0, x)=u_{0}(x), x \in \mathbb{R}^{n}$. Then we have that

$$
u(T, x)=\left(e^{t \triangle} u_{0}\right)(x) \text { for all } x \in \mathbb{R}^{n} .
$$

Given $N>0$, write respectively $\chi_{\leq N}(D)$ and $\chi_{>N}(D)$ for the Fourier multipliers with the symbols $\chi_{|\xi| \leq N}$ and $\chi_{|\xi|>N}$. Namely,

$$
\widehat{\chi \leq N}(D) g(\xi):=\chi_{|\xi| \leq N} \widehat{g}(\xi) \text { and } \widehat{\chi>N(D)} g(\xi):=\chi_{|\xi|>N} \widehat{g}(\xi) \text { for all } g \in L^{2}\left(\mathbb{R}^{n}\right),
$$

Then we can express $u_{0}$ as:

$$
u_{0}=\chi_{\leq N}(D) u_{0}+\chi_{>N}(D) u_{0} .
$$

From this and (1.10), we can easily check that

$$
\begin{aligned}
& \int_{\mathbb{R}^{n}}|u(T, x)|^{2} \mathrm{~d} x \\
& \leq 2 \int_{\mathbb{R}^{n}}\left|\left(e^{T \triangle} \chi_{\leq N}(D) u_{0}\right)(x)\right|^{2} \mathrm{~d} x+2 \int_{\mathbb{R}^{n}}\left|\left(e^{T \triangle} \chi_{>N}(D) u_{0}\right)(x)\right|^{2} \mathrm{~d} x \\
& \leq 2 e^{C_{\text {spec }}(1+N)} \int_{E}\left|\left(e^{T \triangle} \chi_{\leq N}(D) u_{0}\right)(x)\right|^{2} \mathrm{~d} x+2 \int_{\mathbb{R}^{n}}\left|\left(e^{T \triangle} \chi_{>N}(D) u_{0}\right)(x)\right|^{2} \mathrm{~d} x
\end{aligned}
$$




$$
\leq 4 e^{C_{s p e c}(1+N)} \int_{E}\left|\left(e^{T \triangle} u_{0}\right)(x)\right|^{2} \mathrm{~d} x+\left(2+4 e^{C_{s p e c}(1+N)}\right) \int_{\mathbb{R}^{n}}\left|\left(e^{T \triangle} \chi_{>N}(D) u_{0}\right)(x)\right|^{2} \mathrm{~d} x .
$$

Since

$$
\begin{aligned}
\int_{\mathbb{R}^{n}}\left|\left(e^{T \triangle} \chi_{>N}(D) u_{0}\right)(x)\right|^{2} \mathrm{~d} x & =\int_{\mathbb{R}^{n}}\left|e^{-T|\xi|^{2}} \chi_{>N}(\xi) \widehat{u_{0}}(\xi)\right|^{2} \mathrm{~d} \xi \\
& \leq e^{-T N^{2}} \int_{\mathbb{R}^{n}}\left|\widehat{u_{0}}(\xi)\right|^{2} \mathrm{~d} \xi \\
& =e^{-T N^{2}} \int_{\mathbb{R}^{n}}\left|u_{0}(x)\right|^{2} \mathrm{~d} x,
\end{aligned}
$$

it follows from (2.31) that

$$
\begin{aligned}
\int_{\mathbb{R}^{n}}|u(T, x)|^{2} \mathrm{~d} x & \leq 4 e^{C_{\text {spec }}(1+N)} \int_{E}|u(T, x)|^{2} \mathrm{~d} x+\left(2+4 e^{C_{\text {spec }}(1+N)}\right) e^{-T N^{2}} \int_{\mathbb{R}^{n}}\left|u_{0}(x)\right|^{2} \mathrm{~d} x \\
& \leq 6 e^{C_{\text {spec }}}\left(e^{C_{\text {spec }} N} \int_{E}|u(T, x)|^{2} \mathrm{~d} x+e^{C_{\text {spec }} N-T N^{2}} \int_{\mathbb{R}^{n}}\left|u_{0}(x)\right|^{2} \mathrm{~d} x\right)
\end{aligned}
$$

Given $\varepsilon \in(0,1)$, choose $N=N(\varepsilon)$ so that

$$
\exp \left[C_{\text {spec }} N-T N^{2}\right]=\varepsilon \text {. }
$$

(This can be done since the set: $\left\{C_{\text {spec }} s-t s^{2}: s>0\right\}$ contains $(-\infty, 0]$.) With the above choice of $N$, we have that

$$
N=\frac{C_{\text {spec }}+\sqrt{C_{\text {spec }}^{2}+4 T \ln \frac{1}{\varepsilon}}}{2 T} \leq \frac{1}{T}\left(C_{\text {spec }}+\sqrt{T \ln \frac{1}{\varepsilon}}\right) .
$$

Thus, with $\theta \in(0,1)$ fixed before, we see that

$$
\begin{aligned}
\exp \left[C_{\text {spec }} N\right] & \leq \exp \left[\frac{C_{\text {spec }}^{2}}{T}\right] \exp \left[\frac{C_{\text {spec }}}{\sqrt{T}} \sqrt{\ln \frac{1}{\varepsilon}}\right] \\
& \leq \exp \left[\frac{C_{\text {spec }}^{2}}{T}\right] \exp \left[\frac{1-\theta}{\theta} \ln \frac{1}{\varepsilon}+\frac{\theta}{1-\theta} \frac{C_{\text {spec }}^{2}}{T}\right] \\
& =\exp \left[\frac{C_{\text {spec }}^{2}}{(1-\theta) T}\right] \varepsilon^{-\frac{1-\theta}{\theta}} .
\end{aligned}
$$

From this and (2.32), we find that for every $\varepsilon \in(0,1)$,

$$
\int_{\mathbb{R}^{n}}|u(T, x)|^{2} \mathrm{~d} x \leq 6 e^{C_{\text {spec }}}\left(e^{\frac{C_{s p e c}^{2}}{(1-\theta) T}} \varepsilon^{-\frac{1-\theta}{\theta}} \int_{E}|u(T, x)|^{2} \mathrm{~d} x+\varepsilon \int_{\mathbb{R}^{n}}\left|u_{0}(x)\right|^{2} \mathrm{~d} x\right) .
$$

Choosing in the above

$$
\varepsilon=\left(\frac{\int_{E}|u(T, x)|^{2} \mathrm{~d} x}{\int_{\mathbb{R}^{n}}\left|u_{0}(x)\right|^{2} \mathrm{~d} x}\right)^{\theta}
$$

we obtain that

$$
\int_{\mathbb{R}^{n}}|u(T, x)|^{2} \mathrm{~d} x \leq 12 e^{C_{s p e c}} e^{\frac{C_{s p e c}^{2}}{(1-\theta) T}}\left(\int_{E}|u(T, x)|^{2} \mathrm{~d} x\right)^{\theta}\left(\int_{\mathbb{R}^{n}}\left|u_{0}(x)\right|^{2} \mathrm{~d} x\right)^{1-\theta}
$$




$$
\leq e^{\left(\frac{1}{1-\theta}\left(C_{\text {spec }}+1\right)^{2}+\ln 12\right)\left(1+\frac{1}{T}\right)}\left(\int_{E}|u(T, x)|^{2} \mathrm{~d} x\right)^{\theta}\left(\int_{\mathbb{R}^{n}}\left|u_{0}(x)\right|^{2} \mathrm{~d} x\right)^{1-\theta},
$$

which leads to (1.7) with

$$
C_{\text {Hold }}=\frac{1}{1-\theta}\left(C_{\text {spec }}+1\right)^{2}+\ln 12 .
$$

This ends the proof of Lemma 2.3.

Lemma 2.4. Suppose that a measurable set $E \subset \mathbb{R}^{n}$ has the property: there is a positive constant $C_{H o l d}=C_{H o l d}(n, E)$ so that for any $T>0$,

$$
\int_{\mathbb{R}^{n}}|u(T, x)|^{2} \mathrm{~d} x \leq e^{C_{H o l d}\left(1+\frac{1}{T}\right)}\left(\int_{E}|u(T, x)|^{2} \mathrm{~d} x\right)^{1 / 2}\left(\int_{\mathbb{R}^{n}}|u(0, x)|^{2} \mathrm{~d} x\right)^{1 / 2},
$$

when $u$ solves the equation (1.1). Then for each $T>0$ and each subset $F \subset(0, T)$ of positive measure, there is a positive constant $C_{o b s}=C_{o b s}\left(n, T, F, C_{H o l d}\right)$ so that when u solves (1.1),

$$
\int_{\mathbb{R}^{n}}|u(T, x)|^{2} \mathrm{~d} x \leq C_{o b s} \int_{F} \int_{E}|u(s, x)|^{2} \mathrm{~d} x d s .
$$

In particular, if $F=(0, T)$ then the constant $C_{o b s}$ in (2.34) can be expressed as:

$$
C_{\text {obs }}=\exp \left[12\left(1+3 C_{\text {Hold }}\right)(1+1 / T)\right] .
$$

Proof. Suppose that $E \subset \mathbb{R}^{n}$ satisfies (2.33). Arbitrarily fix $T>0$ and $F \subset(0, T)$ of positive measure. Applying Cauchy's inequality to (2.33), we find that for all $t>0$ and $\varepsilon>0$

$$
\int_{\mathbb{R}^{n}}|u(t, x)|^{2} \mathrm{~d} x \leq \frac{1}{\varepsilon} e^{2 C_{H o l d}\left(1+\frac{1}{t}\right)} \int_{E}|u(t, x)|^{2} \mathrm{~d} x+\varepsilon \int_{\mathbb{R}^{n}}\left|u_{0}(x)\right|^{2} \mathrm{~d} x .
$$

By a translation in time, we find from (2.35) that for all $0<t_{1}<t_{2}$ and $\varepsilon>0$,

$$
\int_{\mathbb{R}^{n}}\left|u\left(t_{2}, x\right)\right|^{2} \mathrm{~d} x \leq \frac{1}{\varepsilon} e^{2 C_{\text {Hold }}\left(1+\frac{1}{t_{2}-t_{1}}\right)} \int_{E}\left|u\left(t_{2}, x\right)\right|^{2} \mathrm{~d} x+\varepsilon \int_{\mathbb{R}^{n}}\left|u\left(t_{1}, x\right)\right|^{2} \mathrm{~d} x .
$$

Let $l$ be a Lebesgue density point of $F$. Then according to [44, Proposition 2.1], for each $\lambda \in(0,1)$, there is a sequence $\left\{l_{m}\right\}_{l=1}^{\infty} \subset(l, T)$ so that for each $m \in \mathbb{N}^{+}$,

$$
l_{m+1}-l=\lambda^{m}\left(l_{1}-l\right)
$$

and

$$
\left|F \bigcap\left(l_{m+1}, l_{m}\right)\right| \geq \frac{1}{3}\left(l_{m}-l_{m+1}\right) .
$$

Arbitrarily fix $m \in \mathbb{N}^{+}$. Take $s$ so that

$$
0<l_{m+2}<l_{m+1} \leq s<l_{m}<T .
$$

Using (2.36) (with $t_{1}=l_{m+2}$ and $t_{2}=s$ ) and noting that

$$
\int_{\mathbb{R}^{n}}\left|u\left(l_{m}, x\right)\right|^{2} \mathrm{~d} x \leq \int_{\mathbb{R}^{n}}|u(s, x)|^{2} \mathrm{~d} x \text { and } l_{m+1}-l_{m+2} \leq s-l_{m+2},
$$


we see that

$$
\int_{\mathbb{R}^{n}}\left|u\left(l_{m}, x\right)\right|^{2} \mathrm{~d} x \leq \frac{1}{\varepsilon} e^{2 C_{\text {Hold }}\left(1+\frac{1}{l_{m+1}-l_{m+2}}\right)} \int_{E}|u(s, x)|^{2} \mathrm{~d} x+\varepsilon \int_{\mathbb{R}^{n}}\left|u\left(l_{m+2}, x\right)\right|^{2} \mathrm{~d} x .
$$

Integrating with $s$ over $F \bigcap\left(l_{m+1}, l_{m}\right)$ in (2.39) implies that

$$
\begin{array}{rl}
\int_{\mathbb{R}^{n}}\left|u\left(l_{m}, x\right)\right|^{2} & \mathrm{~d} x \leq \varepsilon \int_{\mathbb{R}^{n}}\left|u\left(l_{m+2}, x\right)\right|^{2} \mathrm{~d} x \\
& +\frac{1}{\varepsilon} \frac{1}{\left|F \bigcap\left(l_{m+1}, l_{m}\right)\right|} e^{2 C_{H o l d}\left(1+\frac{1}{l_{m+1}-l_{m+2}}\right)} \int_{F \cap\left(l_{m+1}, l_{m}\right)} \int_{E}|u(s, x)|^{2} \mathrm{~d} x d s .
\end{array}
$$

Since it follows by (2.38) that

$$
\left|F \bigcap\left(l_{m+1}, l_{m}\right)\right| \geq \frac{1}{3}\left(l_{m}-l_{m+1}\right) \geq \frac{1}{3} e^{-\frac{1}{l_{m}-l_{m+1}}},
$$

we obtain from (2.40) that

$$
\begin{aligned}
\int_{\mathbb{R}^{n}}\left|u\left(l_{m}, x\right)\right|^{2} \mathrm{~d} x & \leq \varepsilon \int_{\mathbb{R}^{n}}\left|u\left(l_{m+2}, x\right)\right|^{2} \mathrm{~d} x \\
& +\frac{3}{\varepsilon} e^{\frac{1}{l_{m}-l_{m+1}}+2 C_{\text {Hold }}\left(1+\frac{1}{l_{m+1}-l_{m+2}}\right)} \int_{F \cap\left(l_{m+1}, l_{m}\right)} \int_{E}|u(s, x)|^{2} \mathrm{~d} x d s .
\end{aligned}
$$

Meanwhile, it follows by (2.37) that

$$
l_{m}-l_{m+1}=\frac{1}{1+\lambda}\left(l_{m}-l_{m+2}\right)
$$

and

$$
l_{m+1}-l_{m+2}=\frac{\lambda}{1+\lambda}\left(l_{m}-l_{m+2}\right) .
$$

Inserting (2.42) and (2.43) into (2.41), we find that

$$
\begin{aligned}
\int_{\mathbb{R}^{n}}\left|u\left(l_{m}, x\right)\right|^{2} \mathrm{~d} x & \leq \varepsilon \int_{\mathbb{R}^{n}}\left|u\left(l_{m+2}, x\right)\right|^{2} \mathrm{~d} x \\
& +3 e^{2 C_{H o l d}} \frac{1}{\varepsilon} e^{\frac{C^{\prime}}{l_{m}-l_{m+2}}} \int_{F \cap\left(l_{m+1}, l_{m}\right)} \int_{E}|u(s, x)|^{2} \mathrm{~d} x d s
\end{aligned}
$$

with

$$
C^{\prime}=1+\lambda+\frac{2 C_{\text {Hold }}(1+\lambda)}{\lambda}
$$

Rewrite (2.44) as

$$
\begin{aligned}
& \varepsilon e^{-\frac{C^{\prime}}{l_{m}-l_{m+2}}} \int_{\mathbb{R}^{n}}\left|u\left(l_{m}, x\right)\right|^{2} \mathrm{~d} x-\varepsilon^{2} e^{-\frac{C^{\prime}}{l_{m}-l_{m+2}}} \int_{\mathbb{R}^{n}}\left|u\left(l_{m+2}, x\right)\right|^{2} \mathrm{~d} x \\
\leq & 3 e^{2 C_{\text {Hold }}} \int_{F \cap\left(l_{m+1}, l_{m}\right)} \int_{E}|u(s, x)|^{2} \mathrm{~d} x d s .
\end{aligned}
$$

Next, we fix $\lambda \in(1 / \sqrt{2}, 1)$. Let $\mu:=\frac{1}{2-\lambda^{-2}}$. Then $\mu>1$. Setting, in (2.46),

$$
\varepsilon=\exp \left[-\frac{(\mu-1) C^{\prime}}{l_{m}-l_{m+2}}\right] \text {, }
$$


we have that

$$
\begin{aligned}
& e^{-\frac{\mu C^{\prime}}{l_{m}-l_{m+2}}} \int_{\mathbb{R}^{n}}\left|u\left(l_{m}, x\right)\right|^{2} \mathrm{~d} x-e^{-\frac{(2 \mu-1) C^{\prime}}{l_{m}-l_{m+2}}} \int_{\mathbb{R}^{n}}\left|u\left(l_{m+2}, x\right)\right|^{2} \mathrm{~d} x \\
\leq & 3 e^{2 C_{H o l d}} \int_{F \cap\left(l_{m+1}, l_{m}\right)} \int_{E}|u(s, x)|^{2} \mathrm{~d} x d s .
\end{aligned}
$$

Meanwhile, one can easily check that

$$
\exp \left[-\frac{(2 \mu-1) C^{\prime}}{l_{m}-l_{m+2}}\right]=\exp \left[-\frac{\mu C^{\prime}}{\lambda^{2}\left(l_{m}-l_{m+2}\right)}\right] .
$$

Because

$$
l_{m+2}-l_{m+4}=\lambda^{2}\left(l_{m}-l_{m+2}\right),
$$

we deduce from (2.47) and (2.48) that

$$
\begin{aligned}
& e^{-\frac{\mu C^{\prime}}{l_{m}-l_{m+2}}} \int_{\mathbb{R}^{n}}\left|u\left(l_{m}, x\right)\right|^{2} \mathrm{~d} x-e^{-\frac{\mu C^{\prime}}{l_{m+2} l_{m+4}}} \int_{\mathbb{R}^{n}}\left|u\left(l_{m+2}, x\right)\right|^{2} \mathrm{~d} x \\
\leq & 3 e^{2 C_{\text {Hold }}} \int_{F \cap\left(l_{m+1}, l_{m}\right)} \int_{E}|u(s, x)|^{2} \mathrm{~d} x d s .
\end{aligned}
$$

Summing the above inequality from $m=1$ to $\infty$ derives that

$$
\begin{aligned}
e^{-\frac{\mu C^{\prime}}{l_{1}-l_{3}}} \int_{\mathbb{R}^{n}}\left|u\left(l_{1}, x\right)\right|^{2} \mathrm{~d} x & \leq 3 e^{2 C_{\text {Hold }}} \sum_{m=1}^{\infty} \int_{F \cap\left(l_{m+1}, l_{m}\right)} \int_{E}|u(s, x)|^{2} \mathrm{~d} x d s \\
& \leq 3 e^{2 C_{\text {Hold }}} \int_{F \cap\left(l, l_{1}\right)} \int_{E}|u(s, x)|^{2} \mathrm{~d} x d s \\
& \leq 3 e^{2 C_{\text {Hold }}} \int_{F} \int_{E}|u(s, x)|^{2} \mathrm{~d} x d s .
\end{aligned}
$$

Thus, we have that

$$
\int_{\mathbb{R}^{n}}|u(T, x)|^{2} \mathrm{~d} x \leq \int_{\mathbb{R}^{n}}\left|u\left(l_{1}, x\right)\right|^{2} \mathrm{~d} x \leq 3 e^{2 C_{\text {Hold }}} e^{\frac{\mu C^{\prime}}{l_{1}-l_{3}}} \int_{F} \int_{E}|u(s, x)|^{2} \mathrm{~d} x d s,
$$

which leads to (2.34) with

$$
C_{o b s}=3 \exp \left[2 C_{H o l d}+\frac{\mu C^{\prime}}{l_{1}-l_{3}}\right] .
$$

Finally, in the case when $F=(0, T)$, we set

$$
l_{1}=\frac{2 T}{3}, l=\frac{T}{3} \quad \text { and } \lambda=\sqrt{\frac{2}{3}} .
$$

Then we have that (see $(2.45))$

$$
\mu=2 \text { and } C^{\prime} \leq 2+6 C_{\text {Hold }}
$$

Now, we derive from (2.49) that

$$
\begin{aligned}
\int_{\mathbb{R}^{n}}|u(T, x)|^{2} \mathrm{~d} x & \leq 3 e^{2 C_{\text {Hold }}} e^{\frac{12\left(1+3 C_{\text {Hold }}\right)}{T}} \int_{0}^{T} \int_{E}|u(s, x)|^{2} \mathrm{~d} x d s \\
& \leq e^{12\left(1+3 C_{\text {Hold }}\right)\left(1+\frac{1}{T}\right)} \int_{0}^{T} \int_{E}|u(s, x)|^{2} \mathrm{~d} x d s .
\end{aligned}
$$

This completes the proof of Lemma 2.4. 
The next lemma seems to be new. The key of its proof is the structure of a special solution to the equation(1.1). This structure is based on the heat kernel.

Lemma 2.5. Suppose that a measurable set $E \subset \mathbb{R}^{n}$ satisfies the observability inequality (1.3). Then the set $E$ is $\gamma$-thick at scale $L$ for some $\gamma>0$ and $L>0$.

Proof. Let $E \subset \mathbb{R}^{n}$ be a measurable set satisfying the observability inequality (1.3). Recall that the heat kernel is as:

$$
K(t, x)=(4 \pi t)^{-n / 2} e^{-|x|^{2} / 4 t}, \quad t>0, x \in \mathbb{R}^{n} .
$$

Given $u_{0} \in L^{2}\left(\mathbb{R}^{n}\right)$, the function defined by

$$
(t, x) \longrightarrow \int_{\mathbb{R}^{n}} K(t, x-y) u_{0}(y) \mathrm{d} y, \quad(t, x) \in(0, \infty) \times \mathbb{R}^{n},
$$

is a solution to the equation (1.1) with the initial condition $u(0, x)=u_{0}(x), x \in \mathbb{R}^{n}$.

Arbitrarily fix $x_{0} \in \mathbb{R}^{n}$. By taking

$$
u_{0}(x)=(4 \pi)^{-n / 2} e^{-\left|x-x_{0}\right|^{2} / 4}, \quad x \in \mathbb{R}^{n},
$$

in (2.50), we get the following solution to the equation (1.1):

$$
v(t, x)=(4 \pi(t+1))^{-\frac{n}{2}} e^{-\frac{\left|x-x_{0}\right|^{2}}{4(t+1)}}, \quad t \geq 0, x \in \mathbb{R}^{n} .
$$

From (2.51), we obtain by direct computations that

$$
\int_{\mathbb{R}^{n}}|v(1, x)|^{2} \mathrm{~d} x=4^{-n} \pi^{-\frac{n}{2}}
$$

From (2.51), we also find that for an arbitrarily fixed $L>0$,

$$
v(t, x) \leq(4 \pi)^{-\frac{n}{2}} e^{-\frac{L^{2}}{16}} e^{-\frac{\left|x-x_{0}\right|^{2}}{16}} \text { for all } 0 \leq t \leq 1 \text { and } x \in \mathbb{R}^{n} \text {, with }\left|x-x_{0}\right| \geq L .
$$

By (2.53), the above solution $v$ satisfies that

$$
\int_{0}^{1} \int_{\left|x-x_{0}\right| \geq L}|v(t, x)|^{2} \mathrm{~d} x \mathrm{~d} t \leq(2 \pi)^{-\frac{n}{2}} e^{-\frac{L^{2}}{8}} .
$$

Meanwhile, by taking $T=1$ and $u=v$ in the observability inequality (1.3), we see that

$$
\int_{\mathbb{R}^{n}}|v(1, x)|^{2} \mathrm{~d} x \leq C \int_{0}^{1} \int_{E}|v(t, x)|^{2} \mathrm{~d} x \mathrm{~d} t .
$$

Here and in what follows, $C$ stands for the constant $C_{o b s}(n, 1, E)$ in $(1.3)$.

Now, it follows from $(2.52),(2.55)$ and (2.54) that

$$
4^{-n} \pi^{-\frac{n}{2}} \leq C \int_{0}^{1} \int_{E \cap B_{L}\left(x_{0}\right)}|v(t, x)|^{2} \mathrm{~d} x \mathrm{~d} t+C(2 \pi)^{-\frac{n}{2}} e^{-\frac{L^{2}}{8}} .
$$

Choose $L>0$ in such a way that

$$
C(2 \pi)^{-\frac{n}{2}} e^{-\frac{L^{2}}{8}} \leq \frac{1}{2} 4^{-n} \pi^{-\frac{n}{2}}
$$


Then by (2.56), we obtain that

$$
\begin{aligned}
\frac{1}{2} 4^{-n} \pi^{-\frac{n}{2}} & \leq C \int_{0}^{1} \int_{E \cap B_{L}\left(x_{0}\right)}|v(t, x)|^{2} \mathrm{~d} x \mathrm{~d} t \\
& =C \int_{0}^{1} \int_{E \cap B_{L}\left(x_{0}\right)}(4 \pi(t+1))^{-n} e^{-\frac{\left|x-x_{0}\right|^{2}}{2(t+1)}} \mathrm{d} x \mathrm{~d} t \\
& \leq C \int_{0}^{1} \int_{E \cap B_{L}\left(x_{0}\right)}(4 \pi)^{-n} \mathrm{~d} x \mathrm{~d} t \leq C(4 \pi)^{-n}\left|E \bigcap B_{L}\left(x_{0}\right)\right|
\end{aligned}
$$

from which, it follows that

$$
\left|E \bigcap B_{L}\left(x_{0}\right)\right| \geq(2 C)^{-1} \pi^{\frac{n}{2}} .
$$

Since $B_{L}\left(x_{0}\right) \subset\left(x_{0}+2 L Q\right)$, we see from (2.57) that

$$
\left|E \bigcap\left(x_{0}+2 L Q\right)\right| \geq(2 C)^{-1} \pi^{\frac{n}{2}} .
$$

From this, we find that there is $L^{\prime}>0$ and $\gamma>0$ so that

$$
\left|E \bigcap\left(x_{0}+L^{\prime} Q\right)\right| \geq \gamma\left(L^{\prime}\right)^{n} .
$$

Notice that $x_{0}$ in (2.58) was arbitrarily taken from $\mathbb{R}^{n}$. Hence, the set $E$ is $\gamma$-thick at scale $L^{\prime}$. This ends the proof of Lemma 2.5.

We now on the position to prove Theorem 1.1.

Proof of Theorem 1.1. We can prove it in the following way:

$$
\text { (i) } \Rightarrow(\text { ii }) \Rightarrow(\text { iii }) \Rightarrow(\text { iv }) \Rightarrow(\mathbf{i}) \text {. }
$$

Indeed, the conclusions (i) $\Rightarrow$ (ii), (ii) $\Rightarrow$ (iii), (iii) $\Rightarrow$ (iv) and (iv) $\Rightarrow$ (i) follow respectively from Lemma 2.1, Lemma 2.3, Lemma 2.4, and Lemma 2.5. This ends the proof of Theorem 1.1.

Tracking the constants in Lemma 2.1, Lemma 2.3 and Lemma 2.4, we can easily get the following consequences of Theorem 1.1:

Corollary 2.1. Let $E \subset \mathbb{R}^{n}$ be a set of $\gamma$-thick at scale $L$ for some $\gamma>0$ and $L>0$. Then the following conclusions are true for a constant $C=C(n)>0$ :

(a) The set $E$ satisfies the Hölder-type interpolation (1.7) with

$$
C_{\text {Hold }}(n, E, \theta)=\frac{C}{1-\theta}(1+L)^{2}\left(1+\ln \frac{1}{\gamma}\right)^{2} .
$$

(b) The set $E$ satisfies the observability inequality (1.3) with

$$
C_{\text {obs }}(n, E, T)=e^{96 C(1+L)^{2}\left(1+\ln \frac{1}{\gamma}\right)^{2}\left(1+\frac{1}{T}\right)} .
$$




\section{$3 \quad$ Weak interpolation and observability inequalities}

In this section, we introduce several weak observability inequalities and interpolation inequalities, where observations are made over a ball in $\mathbb{R}^{n}$. One one hand, these inequalities can be viewed as extensions of (1.3) and (1.7) in some senses, while on the other hand, they are independently interesting.

\subsection{Weak interpolation inequalities with observation on the unit ball}

We begin with introducing two spaces. Given $a>0$ and $\nu>0$, we set

$$
L^{2}\left(e^{a|x|^{\nu}} \mathrm{d} x\right):=\left\{f: \mathbb{R}^{n} \rightarrow \mathbb{R} \mid f \text { is measurable and }\|f\|_{L^{2}\left(e^{a|x|^{\nu}} \mathrm{d} x\right)}<+\infty\right\},
$$

equipped with the norm:

$$
\|f\|_{L^{2}\left(e^{a|x|^{\nu}} \mathrm{d} x\right)}:=\left(\int_{\mathbb{R}^{n}}|f(x)|^{2} e^{a|x|^{\nu}} \mathrm{d} x\right)^{1 / 2}, f \in L^{2}\left(e^{a|x|^{\nu}} \mathrm{d} x\right) .
$$

Given $\nu>0$, we set

$$
L^{2}\left(\langle x\rangle^{\nu} \mathrm{d} x\right):=\left\{f: \mathbb{R}^{n} \rightarrow \mathbb{R} \mid f \text { is measurable and }\|f\|_{L^{2}\left(\langle x\rangle^{\nu} \mathrm{d} x\right)}<+\infty\right\},
$$

equipped with the norm:

$$
\|f\|_{L^{2}\left(\langle x\rangle^{\nu} \mathrm{d} x\right)}:=\left(\int_{\mathbb{R}^{n}}|f(x)|^{2}\langle x\rangle^{\nu} \mathrm{d} x\right)^{1 / 2}, \quad f \in L^{2}\left(\langle x\rangle^{\nu} \mathrm{d} x\right) .
$$

In this subsection, we will build up some interpolation inequalities for solutions to (1.1), with initial data in $L^{2}\left(e^{a|x|^{\nu}} \mathrm{d} x\right)$ (or $\left.L^{2}\left(\langle x\rangle^{\nu} \mathrm{d} x\right)\right)$. In these inequalities, observations are made over the unit ball in $\mathbb{R}^{n}$ and at one time point. The purpose to study such observability has been explained in Subsection 1.3. Our main results about this subject are included in the following theorem:

Theorem 3.1. (i) Let $T>0$ and $a>0$. Then there is a constant

$$
C_{1}(a, T)=e^{C^{\prime}\left(1+\frac{1}{T}+a+a^{2} T\right)} \sqrt{\left(1+a^{-n} \Gamma\left(\frac{a}{2|\ln \theta|}\right)\right)} \text {, with } C^{\prime}=C^{\prime}(n)>0, \theta=\theta(n) \in(0,1),
$$

so that for any $\varepsilon>0$,

$$
\int_{\mathbb{R}^{n}}|u(T, x)|^{2} \mathrm{~d} x \leq C_{1}(a, T)\left(\varepsilon \int_{\mathbb{R}^{n}}\left|u_{0}(x)\right|^{2} e^{a|x|} \mathrm{d} x+\varepsilon^{-1} e^{\varepsilon^{-\frac{4|\ln \theta|}{a}}} \int_{B_{1}}|u(T, x)|^{2} \mathrm{~d} x\right),
$$

when $u$ solves (1.1) with the initial condition $u(0, \cdot)=u_{0}(\cdot) \in L^{2}\left(e^{a|x|} \mathrm{d} x\right)$.

(ii) Let $T>0$ and $0<\nu \leq 1$. Then there are two constants

$$
\theta=\theta(n) \in(0,1) \text { and } C_{2}(\nu, T)=\left(1+T^{\frac{\nu}{2}}\right) e^{C^{\prime \prime}\left(1+\frac{1}{T}\right)} \text {, with } C^{\prime \prime}=C^{\prime \prime}(n)
$$

so that for any $\varepsilon \in(0,1)$,

$$
\int_{\mathbb{R}^{n}}|u(T, x)|^{2} \mathrm{~d} x \leq C_{2}(\nu, T)\left(\varepsilon \int_{\mathbb{R}^{n}}\left|u_{0}\right|^{2}\langle x\rangle^{\nu} \mathrm{d} x+e^{e^{(3|\ln \theta|+1)\left(\frac{1}{\varepsilon}\right)^{\frac{1}{\nu}}}} \int_{B_{1}}|u(T, x)|^{2} \mathrm{~d} x\right),
$$

when $u$ solves (1.1) with the initial condition $u(0, \cdot)=u_{0}(\cdot) \in L^{2}\left(\langle x\rangle^{\nu} \mathrm{d} x\right)$. 
Remark 3.1. (a) The condition that $\nu \leq 1$ in (ii) of Theorem 3.1 is not necessary. We make this assumption only for the brevity of the statement of the theorem. Indeed, from the definition of $L^{2}\left(\langle x\rangle^{\nu} \mathrm{d} x\right)$, we see that $L^{2}\left(\langle x\rangle^{\nu} \mathrm{d} x\right) \hookrightarrow L^{2}(\langle x\rangle \mathrm{d} x)$ for any $\nu \geq 1$. From this and (ii) of Theorem 3.1, one can easily check that when $\nu>1$, any solution of (1.1) satisfies that

$$
\int_{\mathbb{R}^{n}}|u(T, x)|^{2} \mathrm{~d} x \leq\left(1+T^{\frac{1}{2}}\right) e^{C^{\prime \prime}(n)\left(1+\frac{1}{T}\right)}\left(\varepsilon \int_{\mathbb{R}^{n}}\left|u_{0}\right|^{2}\langle x\rangle^{\nu} \mathrm{d} x+e^{e^{(3|\ln \theta|+1) \frac{1}{\varepsilon}}} \int_{B_{1}}|u(T, x)|^{2} \mathrm{~d} x\right) .
$$

(b) [15, Theorem 1] contains the following result: There is a universal constant $C>0$ so that for each $T>0$ and $R>0$,

$$
\sup _{0 \leq t \leq T}\left\|e^{\frac{t|x|^{2}}{4\left(t^{2}+R^{2}\right)}} u(t)\right\|_{L^{2}\left(\mathbb{R}^{n}\right)} \leq C\left(\|u(0)\|_{L^{2}\left(\mathbb{R}^{n}\right)}+\left\|e^{\frac{T|x|^{2}}{4\left(T^{2}+R^{2}\right)}} u(T)\right\|_{L^{2}\left(\mathbb{R}^{n}\right)}\right),
$$

when $u$ solves (1.1). The first inequality in Theorem 3.1 is comparable to the above inequality (3.1). By our understanding, these two inequalities can be viewed as different versions of Hardy uncertainty principle. On one hand, the inequality (3.1) can be understood as follows: From some information on a solution to (1.1) at infinity in $\mathbb{R}^{n}$ at two time points 0 and $T$, one can know the behaviour of this solution at infinity in $\mathbb{R}^{n}$ at each time $t \in[0, T]$. On the other hand, the first inequality in Theorem 3.1 can be explained in the following way: From some information on a solution to (1.1) at infinity in $\mathbb{R}^{n}$ at time 0 , and in the ball $B_{1}$ in $\mathbb{R}^{n}$ at time $T$, one can know the behaviour of this solution at infinity in $\mathbb{R}^{n}$ at time $T$.

Similarly, we can compare the second inequality in Theorem 3.1 with (3.1). It deserves to mention that we can only prove inequalities in Theorem 3.1 for the pure heat equation (1.1), while [15, Theorem 1] gave the inequality (3.1) for heat equations with general potentials.

(c) The first inequality in Theorem 3.1 can also be understood as follows: If we know in advance that the initial datum of a solution to (1.1) is in the unit ball of $L^{2}\left(e^{a|x|} \mathrm{d} x\right)$, then we can approximately recover this solution over $\mathbb{R}^{n}$ at the same time $T$, with the error $C_{1}(a, T) \varepsilon$, through observing this solution in the unit ball of $\mathbb{R}^{n}$ at time $T$. The second inequality in Theorem 3.1 can be explained in a very similar way.

To show Theorem 3.1, we need some preliminaries. We begin with some auxiliary lemmas on the persistence of the heat semigroup in the spaces $L^{2}\left(e^{a|x|^{\nu}} \mathrm{d} x\right)$ and $L^{2}\left(\langle x\rangle^{\nu} \mathrm{d} x\right)$.

Lemma 3.1. Let $a>0$ and $0<\nu \leq 1$. Then when $u_{0} \in L^{2}\left(e^{a|x|^{\nu}} \mathrm{d} x\right)$,

$$
\left\|e^{t \triangle} u_{0}\right\|_{L^{2}\left(e^{a|x|^{\nu}} \mathrm{d} x\right)} \leq 2^{\frac{n}{2}} e^{a^{\frac{2}{2-\nu}} t^{\frac{\nu}{2-\nu}}}\left\|u_{0}\right\|_{L^{2}\left(e^{a|x|^{\nu}} \mathrm{d} x\right)} \quad \text { for all } t>0 .
$$

Proof. Arbitrarily fix $a>0,0<\nu \leq 1$ and $u_{0} \in L^{2}\left(e^{a|x|^{\nu}} \mathrm{d} x\right)$. Using the fundamental solution of (1.1) and the definition of $L^{2}\left(e^{a|x|^{\nu}} \mathrm{d} x\right)$, we have that

$$
\left\|e^{t \triangle} u_{0}\right\|_{L^{2}\left(e^{a|x|^{\nu}} \mathrm{d} x\right)}=\left(\int_{\mathbb{R}^{n}}\left(e^{\frac{a|x|^{\nu}}{2}}(4 \pi t)^{-n / 2} \int_{\mathbb{R}^{n}} e^{-\frac{|x-y|^{2}}{4 t}} u_{0}(y) \mathrm{d} y\right)^{2} d x\right)^{\frac{1}{2}}
$$

Since

$$
|x|^{\nu} \leq(|x-y|+|y|)^{\nu} \leq|x-y|^{\nu}+|y|^{\nu} \text { for all } x, y \in \mathbb{R}^{n}
$$

(Here, we used the elementary inequality: $(\tau+s)^{\nu} \leq \tau^{\nu}+s^{\nu}, \tau, s>0$.) it follows from (3.2) that

$$
\left\|e^{t \triangle} u_{0}\right\|_{L^{2}\left(e^{a|x|^{\nu}} \mathrm{d} x\right)} \leq\left(\int_{\mathbb{R}^{n}}\left((4 \pi t)^{-n / 2} \int_{\mathbb{R}^{n}} e^{-\frac{|x-y|^{2}}{4 t}+\frac{a|x-y|^{\nu}}{2}} e^{\frac{a|y|^{\nu}}{2}} u_{0}(y) \mathrm{d} y\right)^{2} d x\right)^{\frac{1}{2}}
$$




$$
\begin{aligned}
& \leq \int_{\mathbb{R}^{n}}(4 \pi t)^{-n / 2} e^{-\frac{|x|^{2}}{4 t}+\frac{a|x|^{\nu}}{2}} d x \cdot\left(\int_{\mathbb{R}^{n}}\left(e^{\frac{a|y|^{\nu}}{2}} u_{0}(y)\right)^{2} d y\right)^{\frac{1}{2}} \\
& =\int_{\mathbb{R}^{n}}(4 \pi t)^{-n / 2} e^{\frac{1}{4 t}\left(-|x|^{2}+2 t a|x|^{\nu}\right)} d x \cdot\left\|u_{0}\right\|_{L^{2}\left(e^{a|x|^{\nu}} \mathrm{d} x\right) .}
\end{aligned}
$$

Meanwhile, by the Young inequality:

$$
2 t a|x|^{\nu} \leq \frac{|x|^{2}}{\frac{2}{\nu}}+\frac{(2 t a)^{\frac{2}{2-\nu}}}{\frac{2}{2-\nu}} \leq \frac{1}{2}|x|^{2}+(2 t a)^{\frac{2}{2-\nu}}
$$

we obtain that

$$
\begin{aligned}
\int_{\mathbb{R}^{n}}(4 \pi t)^{-n / 2} e^{\frac{1}{4 t}\left(-|x|^{2}+2 t a|x|^{\nu}\right)} d x & \leq \int_{\mathbb{R}^{n}} e^{\frac{(2 t a)^{\frac{2}{2-\nu}}}{4 t}}(4 \pi t)^{-n / 2} e^{-\frac{|x|^{2}}{8 t}} d x \\
& =2^{\frac{n}{2}} e^{\frac{(2 t a)}{4 t}} \leq 2^{\frac{2}{2-\nu}} e^{a^{\frac{2}{2-\nu}} t^{\frac{\nu}{2-\nu}}} .
\end{aligned}
$$

Now, the desired inequality follows from (3.3) and (3.4). This ends the proof of Lemma 3.1.

Remark 3.2. The inequality in Lemma 3.1 does not hold for the case when $\nu>1$. Indeed, given $\nu>1$, let $u_{0}(x)=e^{-\frac{1}{2}|x|^{\nu}}\langle x\rangle^{-n}, x \in \mathbb{R}^{n}$. It is clear that $u_{0} \in L^{2}\left(e^{|x|^{\nu}} \mathrm{d} x\right)$. However, we have that for any $t>0, e^{t \triangle} u_{0} \notin L^{2}\left(e^{|x|^{\nu}} \mathrm{d} x\right)$. This can be proved as follows: Arbitrarily fix $t>0$. By some direct calculations, we find that when $|x| \geq 2$,

$$
\left(e^{t \triangle} u_{0}\right)(x) \geq C(4 \pi t)^{-n / 2} e^{-\frac{1}{4 t}} e^{-\frac{1}{2}\left(|x|-\frac{1}{2}\right)^{\nu}}\langle|x|-1 / 2\rangle^{-n} \text { for some } C=C(n) .
$$

This leads to that

$$
\left\|e^{t \triangle} u_{0}\right\|_{L^{2}\left(e^{|x|^{\nu}} \mathrm{d} x\right)} \geq C(4 \pi t)^{-n / 2} e^{-\frac{1}{4 t}}\left(\int_{|x| \geq 2} e^{|x|^{\nu}-\left(|x|-\frac{1}{2}\right)^{\nu}}\langle|x|-1 / 2\rangle^{-2 n} \mathrm{~d} x\right)^{1 / 2} .
$$

Meanwhile, one can easily find a constant $M>2$ so that

$$
|x|^{\nu}-(|x|-1 / 2)^{\nu} \geq \frac{\nu}{4}|x|^{\nu-1}, \text { when }|x| \geq M .
$$

From this and (3.5), we obtain that

$$
\left\|e^{t \triangle} u_{0}\right\|_{L^{2}\left(e^{|x|^{\nu}} \mathrm{d} x\right)} \geq C(4 \pi t)^{-n / 2} e^{-\frac{1}{4 t}}\left(\int_{|x| \geq M} e^{\frac{\nu}{4}|x|^{\nu-1}}\langle|x|-1 / 2\rangle^{-2 n} \mathrm{~d} x\right)^{1 / 2}=\infty .
$$

Lemma 3.2. Let $\nu \geq 0$. Then for any $u_{0} \in L^{2}\left(\langle x\rangle^{\nu} \mathrm{d} x\right)$,

$$
\left\|e^{t \triangle} u_{0}\right\|_{L^{2}\left(\langle x\rangle^{\nu} \mathrm{d} x\right)} \leq 2^{\nu+2} \Gamma(\nu / 2+n)\left(1+t^{\frac{\nu}{4}}\right)\left\|u_{0}\right\|_{L^{2}\left(\langle x\rangle^{\nu} \mathrm{d} x\right)} \text { for all } t>0 .
$$

Proof. The proof is similar to that of Lemma 3.1. (See also [50, Lemma B.6.1].)

Lemma 3.3. Given $s>0$, there is $C=C(n, s)$ so that when $f \in L^{2}\left(\mathbb{R}^{n}\right)$ satisfies that $\widehat{f} \in$ $L^{2}\left(e^{a|\xi|^{s}} \mathrm{~d} \xi\right)$ for some $a>0$,

$$
\left\|D^{\alpha} f\right\|_{L^{\infty}\left(\mathbb{R}^{n}\right)} \leq C^{|\alpha|+1} a^{-\frac{2|\alpha|+3 n}{2 s}}(\alpha !)^{\frac{1}{s}}\|\widehat{f}(\xi)\|_{L^{2}\left(e^{a|\xi| s} \mathrm{~d} \xi\right)} \text { for each } \alpha \in \mathbb{N}^{n} .
$$

(Here, we adopt the convention that $\alpha !=\alpha_{1} ! \alpha_{2} ! \cdots \alpha_{n} !$.) 
Remark 3.3. From Lemma 3.3, we see that if $f \in L^{2}\left(\mathbb{R}^{n}\right)$ satisfies that $\widehat{f} \in L^{2}\left(e^{a|\xi|^{s}} \mathrm{~d} \xi\right)$, with $s>0$ and $a>0$, then $f$ is analytic, when $s=1$, while $f$ is ultra-analytic, when $s>1$.

Proof of Lemma 3.3. Arbitrarily fix $s>0, a>0$ and $f \in L^{2}\left(\mathbb{R}^{n}\right)$, with $\widehat{f} \in L^{2}\left(e^{a|\xi|^{s}} \mathrm{~d} \xi\right)$. Then arbitrarily fix $\alpha=\left(\alpha_{1}, \ldots, \alpha_{n}\right) \in \mathbb{N}^{n}, \beta=\left(\beta_{1}, \ldots, \beta_{n}\right) \in \mathbb{N}^{n}$ and $\gamma=\left(\gamma_{1}, \ldots, \gamma_{n}\right) \in \mathbb{N}^{n}$, with $|\gamma| \leq n$. Several facts are given in order.

Fact One: By direct computations, we see that

$$
\begin{aligned}
\int_{\mathbb{R}^{n}}\left|\xi^{2 \beta}\right| e^{-a|\xi|^{s}} \mathrm{~d} \xi & \leq \int_{\mathbb{R}^{n}}\left|\xi^{2 \beta}\right| e^{-a\left(\sum_{i=1}^{n}\left|\xi_{i}\right|^{s} / n\right)} \mathrm{d} \xi=\prod_{i=1}^{n} \int_{\mathbb{R}_{\xi_{i}}}\left|\xi_{i}\right|^{2 \beta_{i}} e^{-a\left|\xi_{i}\right|^{s} / n} \mathrm{~d} \xi_{i} \\
& =\prod_{i=1}^{n} 2 \int_{0}^{\infty} r^{2 \beta_{i}} e^{-a r^{s} / n} \mathrm{~d} r=\prod_{i=1}^{n} 2\left(\frac{n}{a}\right)^{\frac{2 \beta_{i}+1}{s}} \int_{0}^{\infty} t^{\frac{2 \beta_{i}+1}{s}-1} e^{-t} \mathrm{~d} t \\
& =2^{n}\left(\frac{n}{a}\right)^{\frac{2|\beta|+n}{s}} \prod_{i=1}^{n} \Gamma\left(\frac{2 \beta_{i}+1}{s}\right) .
\end{aligned}
$$

From this, we obtain that

$$
\left(\int_{\mathbb{R}^{n}}\left|\xi^{2(\alpha+\gamma)}\right| e^{-a|\xi|^{s}} \mathrm{~d} \xi\right)^{1 / 2} \leq 2^{n / 2}\left(\frac{n}{a}\right)^{\frac{2|\alpha|+3 n}{2 s}} \prod_{i=1}^{n} \sqrt{\Gamma\left(\frac{2 \alpha_{i}+2 \gamma_{i}+1}{s}\right)} .
$$

Fact Two: By the Sobolev embedding $H^{n}\left(\mathbb{R}^{n}\right) \hookrightarrow L^{\infty}\left(\mathbb{R}^{n}\right)$, we can find $C_{1}(n)>0$ so that

$$
\left\|D^{\alpha} f\right\|_{L^{\infty}\left(\mathbb{R}^{n}\right)} \leq C_{1}(n) \sum_{\gamma \in \mathbb{N}^{n},|\gamma| \leq n}\left\|D^{\alpha+\gamma} f\right\|_{L^{2}\left(\mathbb{R}^{n}\right)} .
$$

Fact Three: By the Plancheral theorem and the Hölder inequality, we obtain that

$$
\left\|D^{\alpha+\gamma} f\right\|_{L^{2}\left(\mathbb{R}^{n}\right)}=\left\|\xi^{\alpha+\gamma} \widehat{f}(\xi)\right\|_{L^{2}\left(\mathbb{R}^{n}\right)} \leq\left\|\widehat{f}(\xi) e^{a|\xi|^{s} / 2}\right\|_{L^{2}\left(\mathbb{R}^{n}\right)}\left(\int_{\mathbb{R}^{n}}\left|\xi^{2(\alpha+\gamma)}\right| e^{-a|\xi|^{s}} \mathrm{~d} \xi\right)^{1 / 2} .
$$

Fact Four: There exists $C_{2}=C_{2}(n, s)$ so that

$$
\sqrt{\Gamma\left(\frac{2 \alpha_{i}+2 \gamma_{i}+1}{s}\right)} \leq C_{2}^{\alpha_{i}+1} \Gamma^{1 / s}\left(\alpha_{i}\right)=C_{2}^{\alpha_{i}+1}\left(\alpha_{i} !\right)^{\frac{1}{s}}
$$

The proof of (3.9) is as follows: From the Stirling formula, we have that

$$
\lim _{x \rightarrow+\infty} \frac{\Gamma(x)}{\sqrt{2 \pi} e^{-x} x^{x+\frac{1}{2}}}=1 .
$$

From (3.10), we can find constants $M_{1}=M_{1}(s)$ and $C_{3}=C_{3}(n, s)$ so that for all $\alpha_{i}>M_{1}$,

$$
\begin{aligned}
\Gamma\left(\frac{2 \alpha_{i}+2 \gamma_{i}+1}{s}\right) & \leq 2 \sqrt{2 \pi} e^{-\frac{2 \alpha_{i}+2 \gamma_{i}+1}{s}}\left(\frac{2 \alpha_{i}+2 \gamma_{i}+1}{s}\right)^{\frac{2 \alpha_{i}+2 \gamma_{i}+1}{s}+\frac{1}{2}} \\
& =2 \sqrt{2 \pi} \cdot e^{-\frac{2 \alpha_{i}+2 \gamma_{i}+1}{s}}\left(\frac{2 \alpha_{i}+2 \gamma_{i}+1}{s}\right)^{\frac{2 \gamma_{i}+1}{s}+\frac{1}{2}} \cdot\left(\frac{2 \alpha_{i}+2 \gamma_{i}+1}{s}\right)^{\frac{2 \alpha_{i}}{s}} \\
& \leq 2 \sqrt{2 \pi} \sup _{x>0} e^{-x} x^{\frac{2 \gamma_{i}+1}{s}+\frac{1}{2}} \cdot\left(\frac{2 \alpha_{i}+2 \gamma_{i}+1}{s}\right)^{\frac{2 \alpha_{i}}{s}} \\
& =2 \sqrt{2 \pi} \cdot e^{-\left(\frac{2 \gamma_{i}+1}{s}+\frac{1}{2}\right)}\left(\frac{2 \gamma_{i}+1}{s}+\frac{1}{2}\right)^{\frac{2 \gamma_{i}+1}{s}+\frac{1}{2}} \cdot\left(\frac{2 \alpha_{i}+2 n+1}{s}\right)^{\frac{2 \alpha_{i}}{s}}
\end{aligned}
$$




$$
\leq C_{3}^{\alpha_{i}} \alpha_{i}^{\frac{2 \alpha_{i}}{s}}
$$

From (3.10), we can also find an absolute constant $M_{2}$ so that for all $\alpha_{i}>M_{2}$,

$$
\Gamma\left(\alpha_{i}\right) \geq 2^{-1} \sqrt{2 \pi} e^{-\alpha_{i}} \alpha_{i}^{\alpha_{i}+\frac{1}{2}} .
$$

According to (3.11) and (3.12), there is a constant $C_{4}(n, s)$ so that

$$
\frac{\sqrt{\Gamma\left(\frac{2 \alpha_{i}+2 \gamma_{i}+1}{s}\right)}}{\Gamma^{1 / s}\left(\alpha_{i}\right)} \leq C_{4}(n, s) \text { for all } \alpha_{i}>M:=\max \left\{M_{1}, M_{2}\right\} .
$$

Meanwhile, it is clear that there is a constant $C_{5}(n, s)$ so that

$$
\frac{\sqrt{\Gamma\left(\frac{2 \alpha_{i}+2 \gamma_{i}+1}{s}\right)}}{\Gamma^{1 / s}\left(\alpha_{i}\right)} \leq C_{5}(n, s) \text { for all } \alpha_{i} \leq M \text {. }
$$

Combining (3.13) and (3.14) leads to (3.9).

Inserting (3.9) into (3.6), noticing that $|\gamma| \leq n$, we find that for some $C_{6}=C_{6}(n, s)$,

$$
\begin{aligned}
\left(\int_{\mathbb{R}^{n}}\left|\xi^{2(\alpha+\gamma)}\right| e^{-a|\xi|^{s}} \mathrm{~d} \xi\right)^{1 / 2} & \leq 2^{n / 2}\left(\frac{n}{a}\right)^{\frac{2|\alpha|+3 n}{2 s}} \prod_{i=1}^{n} C_{2}^{\alpha_{i}+1}\left(\alpha_{i} !\right)^{\frac{1}{s}} \\
& \leq C_{6}^{|\alpha|+1} a^{-\frac{2|\alpha|+3 n}{2 s}}(\alpha !)^{\frac{1}{s}} .
\end{aligned}
$$

Finally, it follows from (3.7), (3.8) and (3.15) that for some $C=C(n, s)$,

$$
\begin{aligned}
\left\|D^{\alpha} f\right\|_{L^{\infty}\left(\mathbb{R}^{n}\right)} & \leq C_{1}(n) \sum_{\gamma \in \mathbb{N}^{n},|\gamma| \leq n}\left\|\widehat{f}(\xi) e^{a|\xi|^{s} / 2}\right\|_{L^{2}\left(\mathbb{R}^{n}\right)} C_{6}^{|\alpha|+1}(\alpha !)^{\frac{1}{s}} \\
& \leq C^{|\alpha|+1} a^{-\frac{2|\alpha|+3 n}{2 s}}(\alpha !)^{\frac{1}{s}}\|\widehat{f}(\xi)\|_{L^{2}\left(e^{a|\xi|^{s}} \mathrm{~d} \xi\right)} .
\end{aligned}
$$

This ends the proof of Lemma 3.3.

The next corollary is a consequence of Lemma 3.3.

Corollary 3.1. There is $C=C(n)>0$ so that when $f \in L^{2}\left(\mathbb{R}^{n}\right)$ satisfies that $\widehat{f} \in L^{2}\left(e^{a|\xi|^{2}} \mathrm{~d} \xi\right)$ for some $a>0$,

$$
\left\|D^{\alpha} f\right\|_{L^{\infty}\left(\mathbb{R}^{n}\right)} \leq e^{C\left(1+b^{2}\right)\left(1+\frac{1}{a}\right)} \frac{|\alpha| !}{b^{|\alpha|}}\|\widehat{f}(\xi)\|_{L^{2}\left(e^{\left.a|\xi|^{2} \mathrm{~d} \xi\right)}\right.} \text { for all } b>0 \text { and } \alpha \in \mathbb{N}^{n} .
$$

Remark 3.4. Let $u_{0} \in L^{2}\left(\mathbb{R}^{n}\right)$ be arbitrarily given. Set $u(t, x)=\left(e^{t \triangle} u_{0}\right)(x),(t, x) \in(0, \infty) \times \mathbb{R}^{n}$. Then $u$ is the solution of (1.1) with $u(0, \cdot)=u_{0}(\cdot)$. Arbitrarily fix $t>0$. By applying Corollary 3.1 (where $f(\cdot)=u(t, \cdot)$ and $a=2 t$ ), we see that the radius of analyticity of $u(t, \cdot)$ (which is treated as a function of $x$ ) is independent $t$. It is an analogy result for solutions of the heat equation in a bounded domain with an analytic boundary (see [2, 16]). This property plays a very important role in the proof of the observability estimates from measurable sets when using the telescope series method developed in [2, 16]. 
Proof of Corollary 3.1. Arbitrarily fix $a>0$ and $f \in L^{2}\left(\mathbb{R}^{n}\right)$ with $\widehat{f} \in L^{2}\left(e^{a|\xi|^{2}} \mathrm{~d} \xi\right)$. Then arbitrarily fix $b>0$ and $\alpha \in \mathbb{N}^{n}$. According to Lemma 3.3 (with $s=2$ ), there is $C^{\prime}=C^{\prime}(n)$ so that

$$
\begin{aligned}
\left\|D^{\alpha} f\right\|_{L^{\infty}\left(\mathbb{R}^{n}\right)} & \leq C^{\prime|\alpha|+1} a^{-\frac{2|\alpha|+3 n}{4}}(\alpha !)^{\frac{1}{2}}\|\widehat{f}(\xi)\|_{L^{2}\left(e^{\left.a|\xi|^{2} \mathrm{~d} \xi\right)}\right.} \\
& \leq g(|\alpha|) \frac{|\alpha| !}{b^{|\alpha|}}\|\widehat{f}(\xi)\|_{L^{2}\left(e^{a|\xi|^{2}} \mathrm{~d} \xi\right)}
\end{aligned}
$$

where

$$
g(r)=C^{\prime} a^{-3 n / 4}\left(b C^{\prime} a^{-1 / 2}\right)^{r}(r !)^{-1 / 2}, r>0 .
$$

To estimate $g(r)$ pointwisely, we use (3.12) to find that when $r>M_{2} \geq 1$ (where $M_{2}$ is given by $(3.12))$,

$$
\begin{aligned}
g(r) & \leq C^{\prime} a^{-3 n / 4}\left(b C^{\prime} a^{-1 / 2}\right)^{r}\left(2^{-1} \sqrt{2 \pi} e^{-r} r^{r+\frac{1}{2}}\right)^{-1 / 2} \\
& \leq 2^{1 / 4} \pi^{-1 / 4} C^{\prime} a^{-3 n / 4}\left(b e^{1 / 2} C^{\prime} a^{-1 / 2}\right)^{r} r^{-r / 2} \\
& \leq 2^{1 / 4} \pi^{-1 / 4} C^{\prime} a^{-3 n / 4} \sup _{r>0}\left(\left(b e^{1 / 2} C^{\prime} a^{-1 / 2}\right)^{r} r^{-r / 2}\right) \\
& =2^{1 / 4} \pi^{-1 / 4} C^{\prime} a^{-3 n / 4} e^{\frac{b^{2} C^{\prime 2}}{2 a}} .
\end{aligned}
$$

Meanwhile, it is clear that when $r \leq M_{2}$,

$$
g(r) \leq C^{\prime} a^{-3 n / 4}\left(b C^{\prime} a^{-1 / 2}+1\right)^{M_{2}} .
$$

From (3.17) and (3.18), it follows that

$$
g(r) \leq e^{C\left(1+b^{2}\right)\left(1+\frac{1}{a}\right)} \text { for all } r>0,
$$

which, together with (3.16), yields the desired inequality. This ends the proof of Corollary 3.1.

To prove Theorem 3.1, we also need the decomposition:

$$
\mathbb{R}^{n}=\bigcup_{j \geq 1} \Omega_{j}, \text { with } \Omega_{j}:=\left\{x \in \mathbb{R}^{n}: j-1 \leq|x| \leq j\right\} .
$$

In the polar coordinate system, we have that

$$
\Omega_{j}=\left\{\left(r, \vartheta_{1}, \cdots, \vartheta_{n-1}\right) \in[j-1, j] \times[0,2 \pi]^{n-1}\right\}, \quad j \geq 1 .
$$

When $j$ is large, the distance between two points in $\Omega_{j}$ can be very large. This makes our studies harder. To pass this barrier, we need a suitable refinement for each $\Omega_{j}$. Given $j \geq 1$, we set

$$
[0,2 \pi]=\bigcup_{1 \leq l \leq j} \Delta_{l}, \text { with } \Delta_{l}:=\left[\frac{l-1}{j} 2 \pi, \frac{l}{j} 2 \pi\right] .
$$

Given $j \geq 1$ and $\left(k_{1}, \ldots, k_{n-1}\right) \in \mathbb{N}^{n-1}$ with $1 \leq k_{i} \leq j$ for all $1 \leq i \leq n-1$, we set

$$
\Omega_{j ; k_{1}, \cdots, k_{n-1}}=\left\{\left(r, \vartheta_{1}, \cdots, \vartheta_{n-1}\right) \in[j-1, j] \times[0,2 \pi]^{n-1}: \vartheta_{1} \in \Delta_{k_{1}}, \cdots, \vartheta_{n-1} \in \Delta_{k_{n-1}}\right\} .
$$


Then one can easily check that for each $j \geq 1$,

$$
\Omega_{j ; k_{1}, \cdots, k_{n-1}} \bigcap \Omega_{j ; k_{1}^{\prime}, \cdots, k_{n-1}^{\prime}}=\emptyset \text {, if }\left(k_{1}, \cdots, k_{n-1}\right) \neq\left(k_{1}^{\prime}, \cdots, k_{n-1}^{\prime}\right),
$$

and

$$
\Omega_{j}=\bigcup \Omega_{j ; k_{1}, \cdots, k_{n-1}},
$$

where the union is taken over all different $\left(k_{1}, \ldots, k_{n-1}\right) \in \mathbb{N}^{n-1}$, with $1 \leq k_{i} \leq j$ for all $i=$ $1, \ldots, n-1$. It should be mentioned that in the constructions of the decompositions (3.19) and (3.24), we borrowed ideas from [24].

Write $\left|\Omega_{j}\right|\left(\left|\Omega_{j ; k_{1}, \cdots, k_{n-1}}\right|\right.$, resp.) for the volume of $\Omega_{j}\left(\Omega_{j ; k_{1}, \cdots, k_{n-1}}\right.$, resp.); Write $d\left(\Omega_{j ; k_{1}, \cdots, k_{n-1}}\right)$ for the diameter of $\Omega_{j ; k_{1}, \cdots, k_{n-1}}$. Then we have the following conclusions:

Conclusion One. There are constants $c_{1}=c_{1}(n)$ and $c_{2}=c_{2}(n)$ so that for any $j \geq 1$ and any $\left(k_{1}, \ldots, k_{n-1}\right) \in \mathbb{N}^{n-1}$, with $1 \leq k_{i} \leq j$ for all $i=1, \ldots, n-1$,

$$
c_{1} V_{n} \leq\left|\Omega_{j ; k_{1}, \cdots, k_{n-1}}\right| \leq c_{2} V_{n} .
$$

Conclusion Two. We have that for any $j \geq 1$ and any $\left(k_{1}, \ldots, k_{n-1}\right) \in \mathbb{N}^{n-1}$, with $1 \leq k_{i} \leq j$ for all $i=1, \ldots, n-1$,

$$
d\left(\Omega_{j ; k_{1}, \cdots, k_{n-1}}\right)=d\left(\Omega_{j ; 1, \cdots, 1}\right):=\sup _{x, x^{\prime} \in \Omega_{j ; 1, \cdots, 1}}\left|x-x^{\prime}\right| .
$$

Conclusion Three. We have that for any fix $j \geq 1$ and $\left(k_{1}, \ldots, k_{n-1}\right) \in \mathbb{N}^{n-1}$, with $1 \leq k_{i} \leq j<$ $j+1$ for all $i=1, \ldots, n-1$,

$$
\begin{gathered}
d\left(\Omega_{j ; k_{1}, \cdots, k_{n-1}}\right) \leq 2 \pi \sqrt{\sum_{1 \leq i \leq n} i^{2}} \leq 2 \pi n^{\frac{3}{2}} ; \\
d\left(\Omega_{j+1 ; k_{1}, \cdots, k_{n-1}}\right) \leq 2 \pi \frac{j+1}{j} \sqrt{\sum_{1 \leq i \leq n} i^{2}} \leq 4 \pi n^{\frac{3}{2}} .
\end{gathered}
$$

We now explain why the above conclusions are true.

To see Conclusion One, we use the definitions of $\Omega_{j+1 ; k_{1}, \cdots, k_{n-1}}$ and $\Omega_{j}$ to find that

$$
\left|\Omega_{j ; k_{1}, \cdots, k_{n-1}}\right|=\frac{1}{j^{n-1}}\left|\Omega_{j}\right|=V_{n} \frac{j^{n}-(j-1)^{n}}{j^{n-1}},
$$

which leads to (3.25).

Conclusion Two follows immediately from the definitions of $\Omega_{j ; k_{1}, \cdots, k_{n-1}}$ and $d\left(\Omega_{j ; k_{1}, \cdots, k_{n-1}}\right)$.

To show (3.27) in Conclusion Three, we let $\left(r, \vartheta_{1}, \cdots, \vartheta_{n-1}\right)$ and $\left(r, \vartheta_{1}^{\prime}, \cdots, \vartheta_{n-1}^{\prime}\right)$ be the polar coordinates of $x=\left(x_{1}, \ldots, x_{n}\right)$ and $x^{\prime}=\left(x_{1}^{\prime}, \ldots, x_{n}^{\prime}\right)$, respectively. Then we have that

$$
x, x^{\prime} \in \Omega_{j ; 1, \cdots, 1} \Longleftrightarrow j-1 \leq r \leq j, \quad 0 \leq \vartheta_{l}, \vartheta_{l}^{\prime} \leq \frac{2 \pi}{j} \text { for all } l=1, \cdots, n-1 .
$$

Notice that the connection between $\left(x_{1}, \ldots, x_{n}\right)$ and $\left(r, \vartheta_{1}, \ldots, \vartheta_{n-1}\right)$ is as:

$$
\left\{\begin{array}{l}
x_{1}=r \cos \vartheta_{1}, \\
x_{2}=r \sin \vartheta_{1} \cos \vartheta_{2}, \\
\cdots \\
x_{n-1}=r \sin \vartheta_{1} \sin \vartheta_{2} \cdots \sin \vartheta_{n-2} \cos \vartheta_{n-1}, \\
x_{n}=r \sin \vartheta_{1} \sin \vartheta_{2} \cdots \sin \vartheta_{n-2} \sin \vartheta_{n-1} .
\end{array}\right.
$$


Then, by the mean value theorem, we have that for some $\zeta \in(0,2 \pi / j)$,

$$
\left|x_{1}-x_{1}^{\prime}\right|=r\left|\cos \vartheta_{1}-\cos \vartheta_{1}^{\prime}\right|=r\left|\sin \zeta \cdot\left(\vartheta_{1}-\vartheta_{1}^{\prime}\right)\right| \leq j|\sin \zeta| \frac{2 \pi}{j} \leq 2 \pi .
$$

By inserting suitable terms and using the mean value theorem, we have that

$$
\left|x_{2}-x_{2}^{\prime}\right| \leq r\left(\left|\sin \vartheta_{1} \cos \vartheta_{2}-\sin \vartheta_{1} \cos \vartheta_{2}^{\prime}\right|+\left|\sin \vartheta_{1} \cos \vartheta_{2}^{\prime}-\sin \vartheta_{1}^{\prime} \cos \vartheta_{2}^{\prime}\right|\right) \leq 2 \pi \cdot 2 .
$$

Similarly, we can verify that

$$
\left|x_{i}-x_{i}^{\prime}\right| \leq 2 \pi \cdot i \text { for all } i=3, \ldots, n .
$$

These, along with (3.26), leads to (3.27).

The inequality (3.28) in Conclusion Three can be proved in the same way. The reason that the factor $\frac{j+1}{j}$ appears in (3.28) is as follows: Since $1 \leq k_{i} \leq j<j+1$ (for all $i=1, \ldots, n-1$ ), we see from the definition of $\Omega_{j+1 ; 1, \cdots, 1}$ that

$$
x, x^{\prime} \in \Omega_{j+1 ; 1, \cdots, 1} \Longleftrightarrow j \leq r \leq j+1,0 \leq \vartheta_{l}, \vartheta_{l}^{\prime} \leq \frac{2 \pi}{j} \text { for all } l=1, \cdots, n-1 .
$$

(The above is comparable with (3.29).)

In summary, we conclude that the above three conclusions are true.

To prove Theorem 3.1, we also need the next lemma concerning with the propagation of for real-analytic functions.

Lemma 3.4. There are constants $C=C(n)>0$ and $\theta=\theta(n) \in(0,1)$ so that for any $a>0$ and $j \geq 1$, and for all $\left(k_{1}, \ldots, k_{n-1}\right) \in \mathbb{N}^{n-1}$, with $1 \leq k_{i} \leq j(i=1, \ldots, n-1)$,

$$
\int_{\Omega_{j+1 ; k_{1}, \ldots, k_{n-1}}}|f|^{2} \mathrm{~d} x \leq e^{C\left(1+\frac{1}{a}\right)}\left(\int_{\Omega_{j ; k_{1}, \ldots, k_{n-1}}}|f|^{2} \mathrm{~d} x\right)^{\theta}\left(\int_{\mathbb{R}^{n}}|\widehat{f}|^{2} e^{a|\xi|^{2}} \mathrm{~d} \xi\right)^{1-\theta}
$$

when $f \in L^{2}\left(\mathbb{R}^{n}\right)$ satisfies that $\widehat{f} \in L^{2}\left(e^{a|\xi|^{2}} \mathrm{~d} \xi\right)$.

The proof of Lemma 3.4 needs Corollary 3.1 and the next lemma which is quoted from [1] (see also [2, Theorem 4]), but is originally from [51].

Lemma 3.5 ([1, Theorem 4] Sec. 3). Let $R>0$ and let $f: B_{2 R} \subset \mathbb{R}^{n} \rightarrow \mathbb{R}$ be real analytic in $B_{2 R}$ verifying

$$
\left|D^{\alpha} f(x)\right| \leq M(\rho R)^{-|\alpha|}|\alpha| \text { !, when } x \in B_{2 R} \text { and } \alpha \in \mathbb{N}^{n}
$$

for some positive numbers $M$ and $\rho \in(0,1]$. Let $\omega \subset B_{R}$ be a subset of positive measure. Then there are two constants $C=C\left(\rho,|\omega| /\left|B_{R}\right|\right)>0$ and $\theta=\theta\left(\rho,|\omega| /\left|B_{R}\right|\right) \in(0,1)$ so that

$$
\|f\|_{L^{\infty}\left(B_{R}\right)} \leq C M^{1-\theta}\left(\frac{1}{|\omega|} \int_{\omega}|f(x)| d x\right)^{\theta} .
$$

We now on the position to show Lemma 3.4.

Proof of Lemma 3.4. Let $a>0$ and $j \geq 1$ be arbitrarily given. Arbitrarily fix $\left(k_{1}, \ldots, k_{n-1}\right) \in$ $\mathbb{N}^{n-1}$, with $1 \leq k_{i} \leq j$ for all $i=1, \ldots, n-1$, and then arbitrarily fix $f \in L^{2}\left(\mathbb{R}^{n}\right)$ with $\widehat{f} \in$ $L^{2}\left(e^{a|\xi|^{2}} \mathrm{~d} \xi\right)$. Since $\Omega_{j ; k_{1}, \cdots, k_{n-1}} \bigcup \Omega_{j+1 ; k_{1}, \cdots, k_{n-1}}$ is connected (see (3.22)), it follows from (3.27) and (3.28) that

$$
d\left(\Omega_{j ; k_{1}, \cdots, k_{n-1}} \bigcup \Omega_{j+1 ; k_{1}, \cdots, k_{n-1}}\right) \leq d\left(\Omega_{j ; k_{1}, \cdots, k_{n-1}}\right)+d\left(\Omega_{j+1 ; k_{1}, \cdots, k_{n-1}}\right) \leq 6 \pi n^{\frac{3}{2}} .
$$


Thus, there exists $\widetilde{x} \in \mathbb{R}^{n}$ such that

$$
\Omega_{j ; k_{1}, \cdots, k_{n-1}} \bigcup \Omega_{j+1 ; k_{1}, \cdots, k_{n-1}} \subset B_{R_{0}}(\widetilde{x}) \text {, with } R_{0}=3 \pi n^{\frac{3}{2}} .
$$

According to Corollary 3.1 where $b=R_{0}$, there is $\widehat{C}=\widehat{C}(n)>0$ such that for all $\alpha \in \mathbb{N}^{n}$,

$$
\left\|D^{\alpha} f\right\|_{L^{\infty}\left(\mathbb{R}^{n}\right)} \leq e^{\widehat{C}\left(1+\frac{1}{a}\right)} \frac{|\alpha| !}{R_{0}^{|\alpha|}}\|\widehat{f}(\xi)\|_{L^{2}\left(e^{\left.a|\xi|^{2} \mathrm{~d} \xi\right)}\right.} .
$$

By (3.31), as well as (3.30), we can apply Lemma 3.5, where

$\rho=1, R=R_{0}, B_{R}=B_{R_{0}}(\widetilde{x}), B_{2 R}=B_{2 R_{0}}(\widetilde{x}), \omega=\Omega_{j ; k_{1}, \cdots, k_{n-1}}, M=e^{\widehat{C}\left(1+\frac{1}{a}\right)}\|\widehat{f}(\xi)\|_{L^{2}\left(e^{\left.a|\xi|^{2} \mathrm{~d} \xi\right)}\right.}$, to find constants $C_{0}=C_{0}(n)>0$ and $\theta=\theta(n) \in(0,1)$ so that

$$
\begin{aligned}
\|f\|_{L^{2}\left(B_{R_{0}}(\widetilde{x})\right)} & \leq C_{0}\|f\|_{L^{2}\left(\Omega_{j ; k_{1}, \cdots, k_{n-1}}\right)}^{\theta}\left(e^{\widehat{C}\left(1+\frac{1}{a}\right)}\|\widehat{f}(\xi)\|_{L^{2}\left(e^{\left.a|\xi|^{2} \mathrm{~d} \xi\right)}\right.}\right)^{1-\theta} \\
& \leq C_{0} e^{\widehat{C}\left(1+\frac{1}{a}\right)}\|f\|_{L^{2}\left(\Omega_{j ; k_{1}, \cdots, k_{n-1}}\right)}^{\theta}\|\widehat{f}(\xi)\|_{L^{2}\left(e^{\left.a|\xi|^{2} \mathrm{~d} \xi\right)}\right.}^{\theta-\theta}
\end{aligned}
$$

(Here, we used (3.25) and a coordinate translation.)

Finally, the desired inequality of the lemma follows from (3.32) and (3.30). This ends the proof of Lemma 3.4.

Based on Lemma 3.4, we can have the next propagation result which will be used later.

Lemma 3.6. There exist constants $C=C(n)>0$ and $\theta=\theta(n) \in(0,1)$ so that for any $a>0$ and $j \geq 1$,

$$
\int_{\Omega_{j+1}}|f|^{2} \mathrm{~d} x \leq j^{n-1} e^{C\left(1+\frac{1}{a}\right)}\left(\int_{B_{1}}|f|^{2} \mathrm{~d} x\right)^{\theta^{j}}\left(\int_{\mathbb{R}^{n}}|\widehat{f}|^{2} e^{a|\xi|^{2}} \mathrm{~d} \xi\right)^{1-\theta^{j}}
$$

when $f \in L^{2}\left(\mathbb{R}^{n}\right)$ satisfies that $\widehat{f} \in L^{2}\left(e^{a|\xi|^{2}} \mathrm{~d} \xi\right)$.

Proof. Arbitrarily fix $a>0$ and $j \geq 1$. And then arbitrarily fix $f \in L^{2}\left(\mathbb{R}^{n}\right)$ with $\widehat{f} \in L^{2}\left(e^{a|\xi|^{2}} \mathrm{~d} \xi\right)$. From (3.23) and (3.24), we see that $\Omega_{j}$ is the disjoint union of all $\Omega_{j ; k_{1}, \cdots, k_{n-1}}$ with different $\left(k_{1}, \ldots, k_{n-1}\right) \in \mathbb{N}^{n-1}$ satisfying $1 \leq k_{i} \leq j$ for all $i=1, \ldots, n-1$. Meanwhile, by (3.20), (3.21) and (3.22), one can also check that $\Omega_{j+1}$ is the disjoint union of all $\Omega_{j+1 ; k_{1}, \cdots, k_{n-1}}$ with different $\left(k_{1}, \ldots, k_{n-1}\right) \in \mathbb{N}^{n-1}$ satisfying $1 \leq k_{i} \leq j$ for all $i=1, \ldots, n-1$. These, along with Lemma 3.4 , yield that for some $C=C(n)>0$ and $\theta=\theta(n)$,

$$
\begin{aligned}
& \int_{\Omega_{j+1}}|f|^{2} \mathrm{~d} x=\sum \int_{\Omega_{j+1 ; k_{1}, \cdots, k_{n-1}}}|f|^{2} \mathrm{~d} x \\
& \leq \sum e^{C\left(1+\frac{1}{a}\right)}\left(\int_{\Omega_{j ; k_{1}, \cdots, k_{n-1}}}|f|^{2} \mathrm{~d} x\right)^{\theta}\left(\int_{\mathbb{R}^{n}} \mid \widehat{f}^{2} e^{a|\xi|^{2}} \mathrm{~d} \xi\right)^{1-\theta} \\
& \leq e^{C\left(1+\frac{1}{a}\right)}\left(\sum \int_{\Omega_{j ; k_{1}, \cdots, k_{n-1}}}|f|^{2} \mathrm{~d} x\right)^{\theta}\left(\sum \int_{\mathbb{R}^{n}}|\widehat{f}|^{2} e^{a|\xi|^{2}} \mathrm{~d} \xi\right)^{1-\theta} \\
& =j^{(n-1)(1-\theta)} e^{C\left(1+\frac{1}{a}\right)}\left(\int_{\Omega_{j}}|f|^{2} \mathrm{~d} x\right)^{\theta}\left(\int_{\mathbb{R}^{n}}|\widehat{f}|^{2} e^{a|\xi|^{2}} \mathrm{~d} \xi\right)^{1-\theta},
\end{aligned}
$$


where the sums are taken over all different $\left(k_{1}, \ldots, k_{n-1}\right) \in \mathbb{N}^{n-1}$ with $1 \leq k_{i} \leq j$ for all $i=$ $1, \ldots, n-1$. (Notice that there are $j^{n-1}$ such $\left(k_{1}, \ldots, k_{n-1}\right)$ ). From (3.33), we can use the induction method to verify that

$$
\begin{aligned}
\int_{\Omega_{j+1}}|f|^{2} \mathrm{~d} x \leq & \left(j(j-1)^{\theta}(j-2)^{\theta^{2}} \cdots 2^{\theta^{j-2}} 1^{\theta^{j-1}}\right)^{(n-1)(1-\theta)} \\
& \times e^{C\left(1+\frac{1}{a}\right)\left(1+\theta+\cdots+\theta^{j-1}\right)}\left(\int_{\Omega_{1}}|f|^{2} \mathrm{~d} x\right)^{\theta^{j}}\left(\int_{\mathbb{R}^{n}}|\widehat{f}|^{2} e^{a|\xi|^{2}} \mathrm{~d} \xi\right)^{1-\theta^{j}} \\
\leq & j^{n-1} e^{\frac{C}{1-\theta}\left(1+\frac{1}{a}\right)}\left(\int_{\Omega_{1}}|f|^{2} \mathrm{~d} x\right)^{\theta^{j}}\left(\int_{\mathbb{R}^{n}}|\widehat{\widehat{f}}|^{2} e^{a|\xi|^{2}} \mathrm{~d} \xi\right)^{1-\theta^{j}} .
\end{aligned}
$$

Since $\Omega_{1}=B_{1}$ and $\theta=\theta(n) \in(0,1)$, the desired conclusion in the lemma follows from (3.34). This ends the proof of Lemma 3.6.

The next proposition plays a very important role in the proof of Theorem 3.1.

Proposition 3.1. There exist constants $C=C(n)>0$ and $\theta=\theta(n) \in(0,1)$ so that for any $a>0, t>0$ and $\varepsilon>0$,

$$
\int_{\mathbb{R}^{n}} e^{-a|x|}|f|^{2} \mathrm{~d} x \leq e^{C\left(1+\frac{1}{t}+a\right)}\left(1+a^{-n} \Gamma\left(\frac{a}{2|\ln \theta|}\right)\right)\left(\varepsilon \int_{\mathbb{R}^{n}}|\widehat{f}|^{2} e^{t|\xi|^{2}} \mathrm{~d} \xi+e^{\varepsilon^{-\frac{2|\ln \theta|}{a}}} \int_{B_{1}}|f|^{2} \mathrm{~d} x\right),
$$

when $f \in L^{2}\left(\mathbb{R}^{n}\right)$ satisfies $\widehat{f} \in L^{2}\left(e^{t|\xi|^{2}} \mathrm{~d} \xi\right)$.

To prove Proposition 3.1, we need the following result quoted from [52]:

Lemma 3.7 ([52, Lemma 3.1]). Let $a>0, b \in(0,1)$ and $\theta \in(0,1)$. Then

$$
\sum_{k=1}^{\infty} b^{\theta^{k}} e^{-a k} \leq \frac{e^{a}}{|\ln \theta|} \Gamma\left(\frac{a}{|\ln \theta|}\right)|\ln x|^{-\frac{a}{|\ln \theta|}} .
$$

Proof of Proposition 3.1. Arbitrarily fix $a>0$ and $t>0$. And then arbitrarily fix $f \in L^{2}\left(\mathbb{R}^{n}\right)$ satisfies $\widehat{f} \in L^{2}\left(e^{t|\xi|^{2}} \mathrm{~d} \xi\right)$. It suffices to show the inequality in Proposition 3.1 for the above fixed $a, t, f$ and any $\varepsilon>0$. Without loss of generality, we can assume that

$$
A:=\int_{\mathbb{R}^{n}}|\widehat{f}|^{2} e^{t|\xi|^{2}} \mathrm{~d} \xi \neq 0 \text { and } B:=\int_{B_{1}}|f|^{2} \mathrm{~d} x \neq 0 .
$$

For otherwise, when $A=0$, we have that $f=0$ over $\mathbb{R}^{n}$, thus the desired inequality is trivial; while when $B=0$, we can use the analyticity of $f$ (which follows from Corollary 3.1) to see that $f=0$ over $\mathbb{R}^{n}$ and then the desired inequality is trivial again.

By (3.19), we have that

$$
\begin{aligned}
\int_{\mathbb{R}^{n}} e^{-a|x|}|f|^{2} \mathrm{~d} x & =\int_{B_{1}} e^{-a|x|}|f|^{2} \mathrm{~d} x+\sum_{j \geq 1} \int_{\Omega_{j+1}} e^{-a|x|}|f|^{2} \mathrm{~d} x \\
& \leq \int_{B_{1}}|f|^{2} \mathrm{~d} x+\sum_{j \geq 1} \int_{\Omega_{j+1}} e^{-a j}|f|^{2} \mathrm{~d} x .
\end{aligned}
$$

We now estimate the last term of (3.36). According to Lemma 3.6, there is $C_{1}=C_{1}(n)>0$ and $\theta=\theta(n) \in(0,1)$ so that

$$
\sum_{j \geq 1} \int_{\Omega_{j+1}} e^{-a j}|f|^{2} \mathrm{~d} x \leq e^{C_{1}\left(1+\frac{1}{t}\right)} \sum_{j \geq 1} j^{n-1} e^{-a j}\left(\int_{B_{1}}|f|^{2} \mathrm{~d} x\right)^{\theta^{j}}\left(\int_{\mathbb{R}^{n}}|\widehat{f}|^{2} e^{t|\xi|^{2}} \mathrm{~d} \xi\right)^{1-\theta^{j}}
$$




$$
\leq e^{C_{1}\left(1+\frac{1}{t}\right)} n !(2 / a)^{n} \sum_{j \geq 1} e^{-\frac{a}{2} j} A(B / A)^{\theta^{j}},
$$

where $A$ and $B$ are given by (3.35). In the proof of (3.37), we used the inequality:

$$
j^{n} e^{-\frac{a}{2} j} \leq n !(2 / a)^{n} \text { for all } j \geq 1 .
$$

Meanwhile, by Lemma 3.7 (with $b=B / A$ ), we have that

$$
\sum_{j \geq 1} e^{-\frac{a}{2} j} A(B / A)^{\theta^{j}} \leq \frac{e^{\frac{a}{2}}}{|\ln \theta|} \Gamma\left(\frac{a}{2|\ln \theta|}\right) A|\ln (B / A)|^{-\frac{a}{2|\ln \theta|}} .
$$

About $A / B$, there are only two possibilities: either $A / B>e$ or $A / B \leq e$.

In the first case when $A / B>e$, we claim that

$$
A|\ln (B / A)|^{-\frac{a}{2|\ln \theta|}} \leq \varepsilon A+e^{\varepsilon^{-\frac{2|\ln \theta|}{a}}} B \text { for all } \varepsilon>0 .
$$

In fact, when $\varepsilon$ satisfies that

$$
A|\ln (B / A)|^{-\frac{a}{2|\ln \theta|}} \leq \varepsilon A,
$$

(3.39) is trivial. One the other hand, when $\varepsilon>0$ satisfies that

$$
A|\ln (B / A)|^{-\frac{a}{2|\ln \theta|}}>\varepsilon A,
$$

we have that

$$
A / B<e^{\varepsilon^{-\frac{2|\ln \theta|}{a}}} .
$$

This, along with the fact that $A / B>e$, yields that

$$
A|\ln (B / A)|^{-\frac{a}{2|\ln \theta|}} \leq A \leq B \cdot A / B \leq e^{\varepsilon^{-\frac{2|\ln \theta|}{a}}} B \text { for all } \varepsilon>0 .
$$

Thus, we have proved (3.39).

Inserting (3.39) into (3.38) leads to

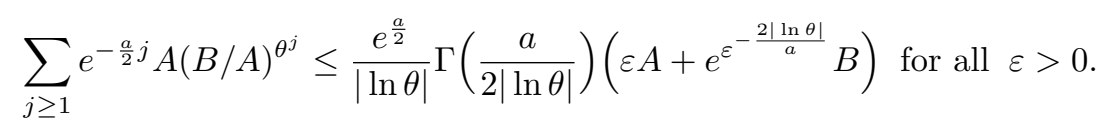

Now it follows from (3.36),(3.37) and (3.40) that for some $C_{2}=C_{2}(n)>0$,

$$
\begin{aligned}
\int_{\mathbb{R}^{n}} e^{-a|x|}|f|^{2} \mathrm{~d} x & \leq e^{C_{1}\left(1+\frac{1}{t}\right)} n !\left(1+\left(\frac{2}{a}\right)^{n} \frac{e^{\frac{a}{2}}}{|\ln \theta|} \Gamma\left(\frac{a}{2|\ln \theta|}\right)\right)\left(\varepsilon A+e^{\varepsilon^{-\frac{2|\ln \theta|}{a}}} B\right) \\
& \leq e^{C_{2}\left(1+\frac{1}{t}+a\right)}\left(1+a^{-n} \Gamma\left(\frac{a}{2|\ln \theta|}\right)\right)\left(\varepsilon A+e^{\varepsilon^{-\frac{2|\ln \theta|}{a}}} B\right) \text { for any } \varepsilon>0 .
\end{aligned}
$$

This proves the desired inequality for the first case that $A / B>e$.

In the second case where $A / B \leq e$, we derive directly that

$$
\begin{aligned}
\int_{\mathbb{R}^{n}} e^{-a|x|}|f|^{2} \mathrm{~d} x & \leq \int_{\mathbb{R}^{n}}|f|^{2} \mathrm{~d} x \leq \int_{\mathbb{R}^{n}}|\widehat{f}|^{2} e^{t|\xi|^{2}} \mathrm{~d} \xi \leq e \int_{B_{1}}|f|^{2} \mathrm{~d} x \\
& \leq e\left(\varepsilon \int_{\mathbb{R}^{n}}|\widehat{f}|^{2} e^{t|\xi|^{2}} \mathrm{~d} \xi+e^{\varepsilon^{-\frac{2|\ln \theta|}{a}}} \int_{B_{1}}|f|^{2} \mathrm{~d} x\right) \text { for any } \varepsilon>0 .
\end{aligned}
$$

This proves the desired inequality for the second case that $A / B \leq e$.

Hence, we end the proof of Proposition 3.1. 
We now are on the position to show Theorem 3.1.

Proof of Theorem 3.1. (i). Arbitrarily fix $u_{0} \in L^{2}\left(e^{a|x|} \mathrm{d} x\right)$. Let $u(T, x)=\left(e^{T \triangle} u_{0}\right)(x), x \in \mathbb{R}^{n}$. By the Hölder inequality, we have that

$$
\int_{\mathbb{R}^{n}}|u(T, x)|^{2} \mathrm{~d} x \leq\left(\int_{\mathbb{R}^{n}}|u(T, x)|^{2} e^{a|x|} \mathrm{d} x\right)^{1 / 2}\left(\int_{\mathbb{R}^{n}}|u(T, x)|^{2} e^{-a|x|} \mathrm{d} x\right)^{1 / 2} .
$$

We will estimate the two terms on right side of (3.41) one by one. For the first term, we apply Lemma 3.1 (with $\nu=1$ ) to obtain that

$$
\int_{\mathbb{R}^{n}}|u(T, x)|^{2} e^{a|x|} \mathrm{d} x \leq 2^{n} e^{2 a^{2} T} \int_{\mathbb{R}^{n}}\left|u_{0}(x)\right|^{2} e^{a|x|} \mathrm{d} x .
$$

To estimate the second term (on right side of (3.41)), we first notice that $\widehat{e^{T \Delta} u_{0}} \in L^{2}\left(e^{T|\xi|^{2}} d \xi\right)$, since $u_{0} \in L^{2}\left(e^{a|x|} \mathrm{d} x\right) \subset L^{2}\left(\mathbb{R}^{n}\right)$. Thus, we can apply Proposition 3.1 (with $f=e^{T \triangle} u_{0}$ and $t=2 T)$ to find $C=C(n)>0$ and $\theta=\theta(n) \in(0,1)$ so that for each $\varepsilon>0$,

$$
\int_{\mathbb{R}^{n}} e^{-a|x|}|u(T, x)|^{2} \mathrm{~d} x \leq C(T, a, n)\left(\varepsilon \int_{\mathbb{R}^{n}}\left|u_{0}(x)\right|^{2} \mathrm{~d} x+e^{\varepsilon^{-\frac{2|\ln \theta|}{a}}} \int_{B_{1}}|u(T, x)|^{2} \mathrm{~d} x\right),
$$

where

$$
C(T, a, n)=e^{C\left(1+\frac{1}{T}+a\right)}\left(1+a^{-n} \Gamma\left(\frac{a}{2|\ln \theta|}\right)\right) .
$$

Inserting (3.42) and (3.43) into (3.41), we get that for each $\varepsilon>0$

$$
\begin{array}{rl}
\int_{\mathbb{R}^{n}} & \left.u(T, x)\right|^{2} \mathrm{~d} x \leq \widehat{C}\left(\int_{\mathbb{R}^{n}}\left|u_{0}(x)\right|^{2} e^{a|x|} \mathrm{d} x\right)^{1 / 2}\left(\varepsilon \int_{\mathbb{R}^{n}}\left|u_{0}(x)\right|^{2} \mathrm{~d} x+e^{\varepsilon^{-\frac{2|\ln \theta|}{a}}} \int_{B_{1}}|u(T, x)|^{2} \mathrm{~d} x\right)^{1 / 2} \\
\leq & \widehat{C}\left(\varepsilon^{1 / 2} \int_{\mathbb{R}^{n}}\left|u_{0}(x)\right|^{2} e^{a|x|} \mathrm{d} x\right)^{1 / 2}\left(\varepsilon^{1 / 2} \int_{\mathbb{R}^{n}}\left|u_{0}(x)\right|^{2} \mathrm{~d} x+\varepsilon^{-1 / 2} e^{\varepsilon^{-\frac{2|\ln \theta|}{a}}} \int_{B_{1}}|u(T, x)|^{2} \mathrm{~d} x\right)^{1 / 2} \\
\leq & 2^{-1} \widehat{C}\left(\varepsilon^{1 / 2} \int_{\mathbb{R}^{n}}\left|u_{0}(x)\right|^{2} e^{a|x|} \mathrm{d} x+\varepsilon^{1 / 2} \int_{\mathbb{R}^{n}}\left|u_{0}(x)\right|^{2} \mathrm{~d} x+\varepsilon^{-1 / 2} e^{\varepsilon^{-\frac{2|\ln \theta|}{a}}} \int_{B_{1}}|u(T, x)|^{2} \mathrm{~d} x\right) \\
\leq & \widehat{C}\left(\varepsilon^{1 / 2} \int_{\mathbb{R}^{n}}\left|u_{0}(x)\right|^{2} e^{a|x|} \mathrm{d} x+\varepsilon^{-1 / 2} e^{\varepsilon^{-\frac{2|\ln \theta|}{a}}} \int_{B_{1}}|u(T, x)|^{2} \mathrm{~d} x\right)
\end{array}
$$

where

$$
\widehat{C}=\widehat{C}(T, a, n)=2^{n / 2} e^{a^{2} T} \sqrt{C(T, a, n)} .
$$

Since $\varepsilon>0$ can be arbitrary taken, we replace $\varepsilon$ by $\varepsilon^{2}$ in (3.44) to get the desired conclusion in (i) of Theorem 3.1.

(ii). Arbitrarily fix $u_{0} \in L^{2}\left(\langle x\rangle^{\nu} \mathrm{d} x\right)$. Let $u(T, x)=\left(e^{T \triangle} u_{0}\right)(x), x \in \mathbb{R}^{n}$. Three facts are given in order. Fact One. Using the inequality:

$$
1 \leq \varepsilon|x|^{\nu}+e^{(1 / \varepsilon)^{\frac{1}{\nu}}} e^{-|x|} \text { for all } \varepsilon>0 \text { and } x \in \mathbb{R}^{n},
$$

we find that

$$
\int_{\mathbb{R}^{n}}|u(T, x)|^{2} \mathrm{~d} x \leq \varepsilon \int_{\mathbb{R}^{n}}|u(T, x)|^{2}|x|^{\nu} \mathrm{d} x+e^{(1 / \varepsilon)^{\frac{1}{\nu}}} \int_{\mathbb{R}^{n}}|u(T, x)|^{2} e^{-|x|} \mathrm{d} x .
$$

Fact Two. Since $0<\nu \leq 1$, we can apply Lemma 3.2 to find $C_{1}=C_{1}(n)$ so that

$$
\int_{\mathbb{R}^{n}}|u(T, x)|^{2}|x|^{\nu} \mathrm{d} x \leq\left(2^{\nu+2} \Gamma(\nu / 2+n)\left(1+T^{\frac{\nu}{4}}\right)\left\|u_{0}\right\|_{L^{2}\left(\langle x\rangle^{\nu} \mathrm{d} x\right)}\right)^{2}
$$




$$
\leq C_{1}\left(1+T^{\frac{\nu}{2}}\right) \int_{\mathbb{R}^{n}}\left|u_{0}(x)\right|^{2}\langle x\rangle^{\nu} \mathrm{d} x .
$$

Fact Three. We can use Proposition 3.1 (with $f=e^{T \triangle} u_{0}, t=2 T$ and $a=1$ ) to find $C_{2}=C_{2}(n)$ and $\theta=\theta(n) \in(0,1)$ so that for all $\mu>0$,

$$
\int_{\mathbb{R}^{n}} e^{-|x|}|u(T, x)|^{2} \mathrm{~d} x \leq e^{C_{2}\left(1+\frac{1}{T}\right)}\left(\mu \int_{\mathbb{R}^{n}}\left|u_{0}(x)\right|^{2} \mathrm{~d} x+e^{\mu^{-2|\ln \theta|}} \int_{B_{1}}\left|\left(e^{T \triangle} u_{0}\right)(x)\right|^{2} \mathrm{~d} x\right) .
$$

To continue the proof, we arbitrarily fix $\varepsilon>0$. We will first use Fact Three, and then use Fact One and Fact Two. By taking $\mu=\varepsilon e^{-\left(\frac{1}{\varepsilon}\right)^{\frac{1}{\alpha}}}$ in (3.47), we obtain that

$$
\begin{aligned}
& e^{(1 / \varepsilon)^{\frac{1}{\nu}}} \int_{\mathbb{R}^{n}} e^{-|x|}|u(T, x)|^{2} \mathrm{~d} x \\
& \leq e^{(1 / \varepsilon)^{\frac{1}{\nu}}} e^{C_{2}\left(1+\frac{1}{T}\right)}\left(\mu \int_{\mathbb{R}^{n}}\left|u_{0}(x)\right|^{2} \mathrm{~d} x+e^{\mu^{-2|\ln \theta|}} \int_{B_{1}}|u(T, x)|^{2} \mathrm{~d} x\right) \\
& =e^{C_{2}\left(1+\frac{1}{T}\right)}\left(\varepsilon \int_{\mathbb{R}^{n}}\left|u_{0}(x)\right|^{2} \mathrm{~d} x+b_{\varepsilon} \int_{B_{1}}|u(T, x)|^{2} \mathrm{~d} x\right),
\end{aligned}
$$

where

$$
b_{\varepsilon}=\exp \left[(1 / \varepsilon)^{\frac{1}{\nu}}+\left(\varepsilon^{-2} e^{2(1 / \varepsilon)^{\frac{1}{\nu}}}\right)^{|\ln \theta|}\right] .
$$

Meanwhile, one can directly check the following two inequalities:

$$
\begin{gathered}
s^{2} \leq e^{s} \text { for all } s>0 ; \\
s+e^{3|\ln \theta| s} \leq e^{(3|\ln \theta|+1) s} \text { for all } s>0 .
\end{gathered}
$$

Choosing $s=\varepsilon^{-1}$ and $s=(1 / \varepsilon)^{\frac{1}{\nu}}$ in (3.49) and (3.50) respectively, using $0<\nu \leq 1$, we find that

$$
\begin{aligned}
b_{\varepsilon} & \leq \exp \left[(1 / \varepsilon)^{\frac{1}{\nu}}+\left(e^{1 / \varepsilon} e^{2(1 / \varepsilon)^{\frac{1}{\nu}}}\right)^{|\ln \theta|}\right] \\
& \leq \exp \left[(1 / \varepsilon)^{\frac{1}{\nu}}+\left(e^{3(1 / \varepsilon)^{\frac{1}{\nu}}}\right)^{|\ln \theta|}\right] \exp \left[(1 / \varepsilon)^{\frac{1}{\nu}}+e^{3|\ln \theta|(1 / \varepsilon)^{\frac{1}{\nu}}}\right] \\
& \leq \exp \left[e^{(3|\ln \theta|+1)(1 / \varepsilon)^{\frac{1}{\nu}}}\right] .
\end{aligned}
$$

Combining (3.48) and (3.51) leads to that

$$
\begin{aligned}
& e^{(1 / \varepsilon)^{\frac{1}{\nu}}} \int_{\mathbb{R}^{n}} e^{-|x|}|u(T, x)|^{2} \mathrm{~d} x \\
& \leq e^{C_{2}(n)\left(1+\frac{1}{T}\right)}\left(\varepsilon \int_{\mathbb{R}^{n}}\left|u_{0}(x)\right|^{2} \mathrm{~d} x+e^{e^{(3|\ln \theta|+1)(1 / \varepsilon) \frac{1}{\nu}}} \int_{B_{1}}|u(T, x)|^{2} \mathrm{~d} x\right) .
\end{aligned}
$$

Finally, inserting (3.46) and (3.52) into (3.45), we obtain that for some $C_{3}=C_{3}(n)$,

$$
\begin{aligned}
& \int_{\mathbb{R}^{n}}|u(T, x)|^{2} \mathrm{~d} x \\
\leq & \left(C_{1}\left(1+T^{\frac{\nu}{2}}\right)+e^{C_{2}\left(1+\frac{1}{T}\right)}\right)\left(\varepsilon \int_{\mathbb{R}^{n}}\left|u_{0}(x)\right|^{2}\langle x\rangle^{\nu} \mathrm{d} x+e^{e^{(3|\ln \theta|+1)(1 / \varepsilon)^{\frac{1}{\nu}}}} \int_{B_{1}}|u(T, x)|^{2} \mathrm{~d} x\right) \\
\leq & \left(1+T^{\frac{\nu}{2}}\right) e^{C_{3}\left(1+\frac{1}{T}\right)}\left(\varepsilon \int_{\mathbb{R}^{n}}\left|u_{0}(x)\right|^{2}\langle x\rangle^{\nu} \mathrm{d} x+e^{e^{(3|\ln \theta|+1)(1 / \varepsilon) \frac{1}{\nu}}} \int_{B_{1}}|u(T, x)|^{2} \mathrm{~d} x\right),
\end{aligned}
$$

which leads to the desired conclusion in (ii) of Theorem 3.1.

Hence, we end the proof of Theorem 3.1. 


\subsection{Weak observability inequalities with observations on balls}

According to Theorem 1.1, it is impossible to recover a solution of (1.1) by observing it over a ball. Thus, two interesting questions arise. First, can we recover a solution of (1.1) over a ball by observing it on another ball? Second, can we have observability inequalities with observations over balls for solutions of (1.1) with some kind of initial values? The answer to the first question is almost negative, while we give partially positive answer for the second question. The first main result of this subsection is stated as follows:

Theorem 3.2. (i) There is an absolute positive constant $C$ so that for all $T>0$ and $0<r^{\prime}<r$,

$$
\int_{B_{r^{\prime}}} u^{2}(T, x) \mathrm{d} x \leq\left(\frac{1}{T}+\frac{C n}{\left(r-r^{\prime}\right)^{2}}\right) \int_{0}^{T} \int_{B_{r}} u^{2}(t, x) \mathrm{d} x \mathrm{~d} t \text {, when u solves }(1.1) .
$$

(ii) Given $T>0$ and $r^{\prime}>r>0$, there is no constant $C=C\left(T, r^{\prime}, r, n\right)$ so that

$$
\int_{B_{r^{\prime}}} u^{2}(T, x) \mathrm{d} x \leq C \int_{0}^{T} \int_{B_{r}} u^{2}(t, x) \mathrm{d} x \mathrm{~d} t \text { for any solution } u \text { to (1.1). }
$$

Proof. (i) Arbitrarily fix $T>0$ and $0<r^{\prime}<r$. Arbitrarily fix a solution $u$ to (1.1). Let $u(0, x)=u_{0}(x), x \in \mathbb{R}^{n}$. Choose a $C^{2}$ function $\varphi$ on $\mathbb{R}^{n}$ so that for some absolute constant $C>0$,

$$
0 \leq \varphi(x) \leq 1 \text { over } \mathbb{R}^{n} ;\left|D^{\alpha} \varphi(x)\right| \leq C\left(r-r^{\prime}\right)^{-|\alpha|} \text { for all } \alpha \in \mathbb{N}^{n}, \text { with }|\alpha| \leq 2,
$$

and so that

$$
\varphi(x)= \begin{cases}1, & x \in B_{r^{\prime}} \\ 0, & x \in B_{r}^{c}\end{cases}
$$

Set $v=\varphi u$. Then $v$ satisfies

$$
v_{t}-\triangle v=-2 \nabla \varphi \cdot \nabla u-\triangle \varphi u \quad \text { in } \mathbb{R}^{+} \times \mathbb{R}^{n}, \quad v(0, \cdot)=\varphi(\cdot) u_{0}(\cdot) \text { in } \mathbb{R}^{n} .
$$

Multiplying (3.54) by $t v$ leads to

$$
\frac{1}{2}\left(t v^{2}\right)_{t}-\frac{1}{2} v^{2}-t v \triangle v=-2 t u \nabla \varphi \cdot \nabla v+t\left(2|\nabla \varphi|^{2}-\varphi \triangle \varphi\right) u^{2} .
$$

Integrating $(3.55)$ over $(0, T) \times \mathbb{R}^{n}$, we have that

$$
\begin{aligned}
& \frac{1}{2} \int_{\mathbb{R}^{n}} T v^{2}(T, x) \mathrm{d} x-\frac{1}{2} \int_{0}^{T} \int_{\mathbb{R}^{n}} v(t, x)^{2} \mathrm{~d} x \mathrm{~d} t+\int_{0}^{T} \int_{\mathbb{R}^{n}} t|\nabla v(t, x)|^{2} \mathrm{~d} x \mathrm{~d} t \\
= & \int_{0}^{T} \int_{\mathbb{R}^{n}}-2 t u \nabla \varphi(x) \cdot \nabla v(t, x) \mathrm{d} x \mathrm{~d} t \\
& +\int_{0}^{T} \int_{\mathbb{R}^{n}} t\left(2|\nabla \varphi(x)|^{2}-\varphi(x) \triangle \varphi(x)\right) u^{2}(t, x) \mathrm{d} x \mathrm{~d} t .
\end{aligned}
$$

Meanwhile, by the Hölder inequality, we find that

$$
\begin{aligned}
& \left|\int_{0}^{T} \int_{\mathbb{R}^{n}}-2 t u(t, x) \nabla \varphi(x) \cdot \nabla v(t, x) \mathrm{d} x \mathrm{~d} t\right| \\
& \leq \int_{0}^{T} \int_{\mathbb{R}^{n}} t|\nabla v(t, x)|^{2} \mathrm{~d} x \mathrm{~d} t+\int_{0}^{T} \int_{\mathbb{R}^{n}} t|\nabla \varphi(x)|^{2} u^{2}(t, x) \mathrm{d} x \mathrm{~d} t .
\end{aligned}
$$


Inserting (3.57) into (3.56) leads to that

$$
\begin{aligned}
& T \int_{\mathbb{R}^{n}} v^{2}(T, x) \mathrm{d} x \\
& \leq \int_{0}^{T} \int_{\mathbb{R}^{n}} v^{2}(t, x) \mathrm{d} x \mathrm{~d} t+2 \int_{0}^{T} \int_{\mathbb{R}^{n}} t\left(3|\nabla \varphi(x)|^{2}-\varphi(x) \triangle \varphi(x)\right) u^{2}(t, x) \mathrm{d} x \mathrm{~d} t .
\end{aligned}
$$

Since $v=u$ on $B_{r^{\prime}}$ and $v=0$ on $B_{r}^{c}$, we can use (3.58) and (3.53) to get that

$$
\begin{aligned}
& \int_{B_{r^{\prime}}} u^{2}(T, x) \mathrm{d} x \\
& \leq \frac{1}{T} \int_{0}^{T} \int_{B_{r}} u^{2}(t, x) \mathrm{d} x \mathrm{~d} t+\frac{2}{T} \int_{0}^{T} \int_{B_{r} \backslash B_{r^{\prime}}} t\left(3|\nabla \varphi(x)|^{2}-\varphi(x) \triangle \varphi(x)\right) u^{2}(t, x) \mathrm{d} x \mathrm{~d} t \\
& \leq\left(\frac{1}{T}+\frac{8 C n}{\left(r-r^{\prime}\right)^{2}}\right) \int_{0}^{T} \int_{B_{r}} u^{2}(t, x) \mathrm{d} x \mathrm{~d} t,
\end{aligned}
$$

which leads to the desired conclusion in (i) of Theorem 3.2.

(ii) By contradiction, we suppose that there were $T>0, r^{\prime}>r>0$ and $C=C\left(T, r^{\prime}, r, n\right)>0$ so that

$$
\int_{B_{r^{\prime}}} u^{2}(T, x) \mathrm{d} x \leq C \int_{0}^{T} \int_{B_{r}} u^{2}(t, x) \mathrm{d} x \mathrm{~d} t \text { for any solution } u \text { to }(1.1) .
$$

We would use a constructive method to derive a contradiction with (3.59). For this purpose, we define, for each $k \geq 1$,

$$
u_{k}(t, x)=\frac{1}{(4 \pi(t+1))^{n / 2}} e^{-\frac{\left|x_{1}-k\right|^{2}+\left|x^{\prime}\right|^{2}}{4(t+1)}}, \quad(t, x)=\left(t, x_{1}, x^{\prime}\right) \in[0, \infty) \times \mathbb{R} \times \mathbb{R}^{n-1} .
$$

One can easily check that $u_{k}$ is the solution of (1.1) with initial value:

$$
u_{k}(0, x)=\frac{1}{(4 \pi)^{n / 2}} e^{-\frac{\left|x_{1}-k\right|^{2}+\left|x^{\prime}\right|^{2}}{4}}, \quad x=\left(x_{1}, x^{\prime}\right) \in \mathbb{R} \times \mathbb{R}^{n-1} .
$$

It is clear that $\left\{u_{k}(0, \cdot)\right\}_{k \geq 1}$ is uniformly bounded in $L^{2}\left(\mathbb{R}^{n}\right)$.

We next show that when $k$ is large enough, $u_{k}$ does not satisfy (3.59) (which leads to a contradiction). To this end, we need two estimates:

$$
\begin{gathered}
\int_{0}^{T} \int_{B_{r}} u_{k}^{2}(t, x) \mathrm{d} x \mathrm{~d} t \leq \frac{r^{n} T V_{n}}{(4 \pi(T+1))^{n}} e^{-\frac{(k-r)^{2}}{2(T+1)}}, \text { when } k \text { is large enough; } \\
\int_{B_{r^{\prime}}} u_{k}^{2}(T, x) \mathrm{d} x \geq \frac{1}{(4 \pi(T+1))^{n}} e^{-\frac{\left(k-r-\frac{\sigma}{3}\right)^{2}}{2(T+1)}}(\sigma / 3)^{n} V_{n}, \text { when } k \text { is large enough. }
\end{gathered}
$$

To show (3.60), we obtain from a direct computation that

$$
\partial_{t}\left(\ln u_{k}(t, x)\right)=-\frac{n}{2} \frac{1}{t+1}+\frac{\left|x_{1}-k\right|^{2}+\left|x^{\prime}\right|^{2}}{4(t+1)^{2}} \text { for all } t \geq 0, x=\left(x_{1}, x^{\prime}\right) \in \mathbb{R}^{n},
$$

from which, it follows that when $t \geq 0$ and $x=\left(x_{1}, x^{\prime}\right) \in \mathbb{R}^{n}$,

$$
\partial_{t}\left(\ln u_{k}(t, x)\right)>0 \Longleftrightarrow\left|x_{1}-k\right|^{2}+\left|x^{\prime}\right|^{2}>2 n(t+1) .
$$


This implies that

$$
k>r+\sqrt{2 n(T+1)} \Longrightarrow \partial_{t}\left(\ln u_{k}(t, x)\right)>0 \text { for all }(t, x) \times(0, T) \times B_{r} .
$$

From the above, we see that when $k>r+\sqrt{2 n(T+1)}$, we have that for each $x \in B_{r}, u_{k}(t, x)$ is an increasing function of $t$ on $[0, T]$. Hence, when $k>r+\sqrt{2 n(T+1)}$,

$$
\begin{aligned}
\int_{0}^{T} \int_{B_{r}} u_{k}^{2}(t, x) \mathrm{d} x \mathrm{~d} t & \leq \int_{0}^{T} \int_{B_{r}} u_{k}^{2}(T, x) \mathrm{d} x \mathrm{~d} t=\int_{0}^{T} \int_{B_{r}} \frac{1}{(4 \pi(T+1))^{n}} e^{-\frac{\left|x_{1}-k\right|^{2}+\left|x^{\prime}\right|^{2}}{2(T+1)}} \mathrm{d} x \mathrm{~d} t \\
& =\frac{T}{(4 \pi(T+1))^{n}} \int_{B_{r}} e^{-\frac{\left|x_{1}-k\right|^{2}+\left|x^{\prime}\right|^{2}}{2(T+1)}} \mathrm{d} x \leq \frac{T}{(4 \pi(T+1))^{n}} \int_{B_{r}} e^{-\frac{|k-r|^{2}}{2(T+1)}} \mathrm{d} x \\
& \leq \frac{r^{n} T V_{n}}{(4 \pi(T+1))^{n}} e^{-\frac{(k-r)^{2}}{2(T+1)}}
\end{aligned}
$$

which leads to (3.60).

We next show (3.61). Given $b>0$, use $B_{r}\left(b, 0^{\prime}\right)$ to denote the ball centered at $(b, 0,0, \cdots, 0)$ and of radius $r$, namely

$$
B_{r}\left(b, 0^{\prime}\right):=\left\{x \in \mathbb{R}^{n}:\left|x_{1}-b\right|^{2}+\left|x^{\prime}\right|^{2} \leq r^{2}\right\} .
$$

Let $k>r^{\prime}$ and $\sigma=r^{\prime}-r$. Then

$$
\begin{aligned}
\int_{B_{r^{\prime}}} u_{k}^{2}(T, x) \mathrm{d} x & \geq \int_{B_{r^{\prime}} \cap B_{k-r-\frac{\sigma}{3}}\left(k, 0^{\prime}\right)} u_{k}^{2}(T, x) \mathrm{d} x \\
& =\int_{B_{r^{\prime}} \cap B_{k-r-\frac{\sigma}{3}}\left(k, 0^{\prime}\right)} \frac{1}{(4 \pi(T+1))^{n}} e^{-\frac{\left|x_{1}-k\right|^{2}+\left|x^{\prime}\right|^{2}}{2(T+1)}} \mathrm{d} x \\
& \geq \frac{1}{(4 \pi(T+1))^{n}} e^{-\frac{\left(k-r-\frac{\sigma}{3}\right)^{2}}{2(T+1)}}\left|B_{r^{\prime}} \bigcap B_{k-r-\frac{\sigma}{3}}\left(k, 0^{\prime}\right)\right| .
\end{aligned}
$$

Meanwhile, it is clear that

$$
B_{\frac{\sigma}{3}}\left(r+2 \sigma / 3,0^{\prime}\right) \subset B_{r^{\prime}} \bigcap B_{k-r-\frac{\sigma}{3}}\left(k, 0^{\prime}\right),
$$

which leads to that

$$
\left|B_{r^{\prime}} \bigcap B_{k-r-\frac{\sigma}{3}}\left(k, 0^{\prime}\right)\right| \geq\left|B_{\frac{\sigma}{3}}\left(r+2 \sigma / 3,0^{\prime}\right)\right|=(\sigma / 3)^{n} V_{n} .
$$

Now (3.61) follows from (3.62) and (3.63) at once.

From (3.60) and (3.61), we find that when $k>\max \left\{r+\sqrt{2 n(T+1)}, r^{\prime}\right\}$,

$$
\begin{aligned}
\frac{\int_{0}^{T} \int_{B_{r}} u_{k}^{2}(t, x) \mathrm{d} x \mathrm{~d} t}{\int_{B_{r^{\prime}}} u_{k}^{2}(T, x) \mathrm{d} x} & \leq \frac{\frac{r^{n} T V_{n}}{(4 \pi(T+1))^{n}} e^{-\frac{(k-r)^{2}}{2(T+1)}}}{\frac{1}{(4 \pi(T+1))^{n}} e^{-\frac{\left(k-r-\frac{\sigma}{3}\right)^{2}}{2(T+1)}}\left(\frac{\sigma}{3}\right)^{n} V_{n}} \\
& =T\left(\frac{3 r}{\sigma}\right)^{n} e^{\frac{1}{2(T+1)}\left(\left(k-r-\frac{\sigma}{3}\right)^{2}-(k-r)^{2}\right)} \\
& =T\left(\frac{3 r}{\sigma}\right)^{n} e^{\frac{1}{2(T+1)}\left(\left(\frac{\sigma}{3}\right)^{2}-\frac{2 \sigma}{3}(k-r)\right)} \\
& =T\left(\frac{3 r}{r^{\prime}-r}\right)^{n} e^{\frac{1}{2(T+1)}\left(\left(\frac{r^{\prime}-r}{3}\right)^{2}-\frac{2 r^{\prime}-r}{3}(k-r)\right) .}
\end{aligned}
$$


Since

$$
\left(\frac{r^{\prime}-r}{3}\right)^{2}-\frac{2 r^{\prime}-r}{3}(k-r) \rightarrow-\infty
$$

we see from (3.64) that

$$
\lim _{k \rightarrow+\infty} \frac{\int_{0}^{T} \int_{B_{r}} u_{k}^{2}(t, x) \mathrm{d} x \mathrm{~d} t}{\int_{B_{r^{\prime}}} u_{k}^{2}(T, x) \mathrm{d} x}=0,
$$

from which, it follows that $u_{k}$ does not satisfy (3.59), when $k$ is large enough. This shows the conclusion in (ii) of Theorem 3.2.

Hence, we end the proof of Theorem 3.2.

The next corollary is a direct consequence of Theorem 3.2.

Corollary 3.2. Given $\nu>0, T>0$ and $r>0$, there is no constant $C=C(T, r, \nu, n)>0$ so that

$$
\int_{\mathbb{R}^{n}} u^{2}(T, x) \rho(x) \mathrm{d} x \leq C \int_{0}^{T} \int_{B_{r}} u^{2}(t, x) \mathrm{d} x \mathrm{~d} t \text { for any } u \text { solves }(1.1)
$$

where either $\rho(x)=\langle x\rangle^{-\nu}, x \in \mathbb{R}^{n}$, or $\rho(x)=e^{-|x|}, x \in \mathbb{R}^{n}$.

Remark 3.5. It was announced in [8, p. 384] (without proof) that given a bounded interval E, there is no positive weight function $\rho$ such that

$$
\int_{0}^{\infty}|u(T, x)|^{2} \rho(x) \mathrm{d} x \leq C \int_{0}^{T} \int_{E}|u(t, x)|^{2} \mathrm{~d} x \mathrm{~d} t
$$

for all solutions of the heat equation in the physical space $(0, \infty)$. The above Corollary 3.2 presents a similar result for the heat equation in the physical space $\mathbb{R}^{n}$.

The second main result of this subsection is stated as follows:

Theorem 3.3. (i) There is a generic constant $C$ so that for any $T>0, M>r>0$ and $u_{0} \in$ $L^{2}\left(\mathbb{R}^{n}\right)$ with supp $u_{0} \subset B_{r}$,

$$
\int_{\mathbb{R}^{n}}|u(T, x)|^{2} \mathrm{~d} x \leq\left(\frac{1}{T}+\frac{C n}{(M-r)^{2}}\right) \int_{0}^{T} \int_{B_{M}}|u(t, x)|^{2} \mathrm{~d} x \mathrm{~d} t,
$$

where $u$ is the solution to (1.1) with $u(0, \cdot)=u_{0}(\cdot)$.

(ii) Assume that $0 \leq u_{0} \in L^{1}\left(\mathbb{R}^{n}\right)$ so that

$$
\int_{B_{r}} u_{0}(x) \mathrm{d} x \geq \mu \int_{\mathbb{R}^{n}} u_{0}(x) \mathrm{d} x \text { for some } r>0 \text { and } \mu \in(0,1) .
$$

Then for any $T>0, M>0$ and any solution $u$ to $(1.1)$ with $u(0, \cdot)=u_{0}(\cdot)$,

$$
\int_{\mathbb{R}^{n}}|u(T, x)|^{2} \mathrm{~d} x \leq \frac{2^{\frac{n}{2}+1} \pi^{\frac{n}{2}} T^{\frac{n}{2}-1}}{V_{n}(r \wedge M)^{n} \mu^{2}} e^{\frac{4 r^{2}}{T}} \int_{0}^{T} \int_{B_{M}}|u(t, x)|^{2} \mathrm{~d} x \mathrm{~d} t .
$$

Here, $r \wedge M:=\min \{r, M\}$. 
Proof. (i) The proof is similar to that of (i) of Theorem 3.2. Arbitrarily fix $T>0, M>r>0$ and $u_{0} \in L^{2}\left(\mathbb{R}^{n}\right)$ with supp $u_{0} \subset B_{r}$. Write $u$ for the solution to $(1.1)$ with $u(0, \cdot)=u_{0}(\cdot)$. Choose a $C^{2}$ function $\varphi$ over $\mathbb{R}^{n}$ so that for some absolute constant $C>0$,

$$
0 \leq \varphi(x) \leq 1 \text { over } \mathbb{R}^{n} ;\left|D^{\alpha} \varphi(x)\right| \leq C\left(r-r^{\prime}\right)^{-|\alpha|} \text { for all } \alpha \in \mathbb{N}^{n} \text {, with }|\alpha| \leq 2
$$

and so that

$$
\varphi(x)= \begin{cases}0, & x \in B_{r} \\ 1, & x \in B_{M}^{c}\end{cases}
$$

Set $v=\varphi u$. Multiplying (3.54) by $v$, we find that

$$
\frac{1}{2}\left(v^{2}\right)_{t}-v \triangle v=-2 u \nabla \varphi \cdot \nabla v+\left(2|\nabla \varphi|^{2}-\varphi \triangle \varphi\right) u^{2}
$$

Integrating (3.67) over $(0, T) \times \mathbb{R}^{n}$, we obtain that

$$
\begin{aligned}
& \frac{1}{2} \int_{\mathbb{R}^{n}} v^{2}(T, x) \mathrm{d} x-\frac{1}{2} \int_{\mathbb{R}^{n}} v^{2}(0, x) \mathrm{d} x+\int_{0}^{T} \int_{\mathbb{R}^{n}}|\nabla v(t, x)|^{2} \mathrm{~d} x \mathrm{~d} t \\
= & \int_{0}^{T} \int_{\mathbb{R}^{n}}-2 u(t, x) \nabla \varphi(x) \cdot \nabla v(t, x) \mathrm{d} x \mathrm{~d} t+\int_{0}^{T} \int_{\mathbb{R}^{n}}\left(2|\nabla \varphi(x)|^{2}-\varphi(x) \triangle \varphi(x)\right) u^{2}(t, x) \mathrm{d} x \mathrm{~d} t .
\end{aligned}
$$

Since the support of $u_{0}$ is contained in $B_{r}$, we have that $v(0, \cdot)=0$ over $\mathbb{R}^{n}$. Then by the Hölder inequality, we deduce from (3.68) that

$$
\int_{\mathbb{R}^{n}} v^{2}(T, x) \mathrm{d} x \leq \int_{0}^{T} \int_{\mathbb{R}^{n}} 2\left(3|\nabla \varphi(x)|^{2}-\varphi(x) \triangle \varphi(x)\right) u^{2}(s, x) \mathrm{d} x \mathrm{~d} s .
$$

Note that (3.69) is still true if we replace $T$ by any $t \in(0, T)$. This implies that

$$
\int_{0}^{T} \int_{\mathbb{R}^{n}} v^{2}(t, x) \mathrm{d} x \mathrm{~d} t \leq \int_{0}^{T} \int_{0}^{t} \int_{\mathbb{R}^{n}} 2\left(3|\nabla \varphi(x)|^{2}-\varphi(x) \triangle \varphi(x)\right) u^{2}(t, x) \mathrm{d} x \mathrm{~d} \tau \mathrm{d} t .
$$

Since $v=u$ on $B_{M}^{c}$, it follows from (3.66) and (3.70) that

$$
\int_{0}^{T} \int_{|x| \geq M} u^{2}(t, x) \mathrm{d} x \leq \frac{C n T}{(M-r)^{2}} \int_{0}^{T} \int_{r \leq|x| \leq M} u^{2}(t, x) \mathrm{d} x \mathrm{~d} t .
$$

Meanwhile, it is clear that

$$
\int_{\mathbb{R}^{n}} u^{2}(T, x) \mathrm{d} x \leq \frac{1}{T} \int_{0}^{T} \int_{\mathbb{R}^{n}} u^{2}(t, x) \mathrm{d} x \mathrm{~d} t .
$$

Combining (3.71) and (3.72), we obtain that

$$
\begin{aligned}
\int_{\mathbb{R}^{n}} u^{2}(T, x) \mathrm{d} x & \leq \frac{1}{T} \int_{0}^{T} \int_{|x| \leq M} u^{2}(t, x) \mathrm{d} x \mathrm{~d} t+\frac{1}{T} \int_{0}^{T} \int_{|x| \geq M} u^{2}(t, x) \mathrm{d} x \mathrm{~d} t \\
& \leq \frac{1}{T} \int_{0}^{T} \int_{|x| \leq M} u^{2}(t, x) \mathrm{d} x \mathrm{~d} t+\frac{1}{T} \frac{C n T}{(M-r)^{2}} \int_{0}^{T} \int_{r \leq|x| \leq M} u^{2}(t, x) \mathrm{d} x \mathrm{~d} t \\
& \leq\left(\frac{1}{T}+\frac{C n}{(M-r)^{2}}\right) \int_{0}^{T} \int_{|x| \leq M} u^{2}(t, x) \mathrm{d} x \mathrm{~d} t .
\end{aligned}
$$


which leads to the conclusion (i) of Theorem 3.3.

(ii) Let $T>0$ and $M>0$ be arbitrarily given. Arbitrarily fix $u_{0}$ so that

$$
0 \leq u_{0} \in L^{1}\left(\mathbb{R}^{n}\right) ; \quad \int_{B_{r}} u_{0}(x) \mathrm{d} x \geq \mu \int_{\mathbb{R}^{n}} u_{0}(x) \mathrm{d} x \text { for some } r>0 \text { and } \mu \in(0,1) .
$$

Write $u$ for the solution to (1.1) with $u(0, \cdot)=u_{0}(\cdot)$.

We first prove that when $0<M \leq r$,

$$
\int_{\mathbb{R}^{n}}|u(T, x)|^{2} \mathrm{~d} x \leq \frac{2^{\frac{n}{2}+1} \pi^{\frac{n}{2}} T^{\frac{n}{2}-1}}{V_{n} M^{n} \mu^{2}} e^{\frac{4 r^{2}}{T}} \int_{0}^{T} \int_{B_{M}}|u(t, x)|^{2} \mathrm{~d} x \mathrm{~d} t .
$$

For this purpose, we need the following two estimates:

$$
\begin{gathered}
\int_{\frac{T}{2}}^{T} \int_{|x| \leq M} u^{2}(t, x) \mathrm{d} x \mathrm{~d} t \geq 2^{-1}(4 \pi)^{-n} V_{n} M^{n} \mu^{2} T^{-(n-1)} e^{-\frac{4 r^{2}}{T}}\left(\int_{\mathbb{R}^{n}} u_{0}(x) \mathrm{d} x\right)^{2} ; \\
\int_{\mathbb{R}^{n}} u^{2}(T, x) \mathrm{d} x \leq 2^{-\frac{3 n}{2}}(\pi T)^{-n / 2}\left(\int_{\mathbb{R}^{n}} u_{0}(x) \mathrm{d} x\right)^{2} .
\end{gathered}
$$

To show (3.75), we observe that

$$
u(t, x)=\int_{\mathbb{R}^{n}}(4 \pi t)^{-n / 2} e^{-\frac{|x-y|^{2}}{4 t}} u_{0}(y) \mathrm{d} y, \quad(t, x) \in(0, \infty) \times \mathbb{R}^{n} .
$$

By (3.77) and (3.73), we find that when $t>0,|x| \leq r$,

$$
\begin{aligned}
u(t, x) & \geq \int_{|y| \leq r}(4 \pi t)^{-n / 2} e^{-\frac{|x-y|^{2}}{4 t}} u_{0}(y) \mathrm{d} y \geq \int_{|y| \leq r}(4 \pi t)^{-n / 2} e^{-\frac{r^{2}}{t}} u_{0}(y) \mathrm{d} y \\
& \geq \mu(4 \pi t)^{-n / 2} e^{-\frac{r^{2}}{t}} \int_{\mathbb{R}^{n}} u_{0}(x) \mathrm{d} x .
\end{aligned}
$$

Since $M \leq r$, it follows from (3.78) that

$$
\begin{aligned}
\int_{\frac{T}{2}}^{T} \int_{|x| \leq M} u^{2}(t, x) \mathrm{d} x \mathrm{~d} t & \geq V_{n} M^{n} \mu^{2}\left(\int_{\mathbb{R}^{n}} u_{0}(x) \mathrm{d} x\right)^{2} \int_{\frac{T}{2}}^{T}(4 \pi t)^{-n} e^{-\frac{2 r^{2}}{t}} \mathrm{~d} t \\
& \geq 2^{-1}(4 \pi)^{-n} V_{n} M^{n} \mu^{2} T^{-(n-1)} e^{-\frac{4 r^{2}}{T}}\left(\int_{\mathbb{R}^{n}} u_{0}(x) \mathrm{d} x\right)^{2},
\end{aligned}
$$

which leads to (3.75).

We now show (3.76). By (3.77) and the Young inequality, we have that

$$
\|u(T, x)\|_{L^{2}\left(\mathbb{R}^{n}\right)} \leq\left\|(4 \pi T)^{-n / 2} e^{-\frac{|x|^{2}}{4 T}}\right\|_{L^{2}\left(\mathbb{R}^{n}\right)} \int_{\mathbb{R}^{n}} u_{0}(x) \mathrm{d} x=2^{-\frac{3 n}{4}}(\pi T)^{-n / 4} \int_{\mathbb{R}^{n}} u_{0}(x) \mathrm{d} x,
$$

which leads to (3.76).

Next, by (3.75) and (3.76), we see that

$$
\int_{\mathbb{R}^{n}} u^{2}(T, x) \mathrm{d} x \leq \frac{2^{\frac{n}{2}+1} \pi^{\frac{n}{2}} T^{\frac{n}{2}-1}}{V_{n} M^{n} \mu^{2}} e^{\frac{4 r^{2}}{T}} \int_{\frac{T}{2}}^{T} \int_{|x| \leq M} u^{2}(t, x) \mathrm{d} x \mathrm{~d} t
$$




$$
\leq \frac{2^{\frac{n}{2}+1} \pi^{\frac{n}{2}} T^{\frac{n}{2}-1}}{V_{n} M^{n} \mu^{2}} e^{\frac{4 r^{2}}{T}} \int_{0}^{T} \int_{|x| \leq M} u^{2}(t, x) \mathrm{d} x \mathrm{~d} t
$$

which leads to (3.74) for the case when $0<M \leq r$.

Finally, when $M>r$, we apply (3.74) (with $M=r$ ) to obtain that

$$
\begin{aligned}
\int_{\mathbb{R}^{n}} u^{2}(T, x) \mathrm{d} x & \leq \frac{2^{\frac{n}{2}+1} \pi^{\frac{n}{2}} T^{\frac{n}{2}-1}}{V_{n} r^{n} \mu^{2}} e^{\frac{4 r^{2}}{T}} \int_{0}^{T} \int_{|x| \leq r} u^{2}(t, x) \mathrm{d} x \mathrm{~d} t \\
& \leq \frac{2^{\frac{n}{2}+1} \pi^{\frac{n}{2}} T^{\frac{n}{2}-1}}{V_{n} r^{n} \mu^{2}} e^{\frac{4 r^{2}}{T}} \int_{0}^{T} \int_{|x| \leq M} u^{2}(t, x) \mathrm{d} x \mathrm{~d} t
\end{aligned}
$$

which leads to (3.74) for the case that $M>r$. So the conclusion (ii) in Theorem 3.3 is true.

Hence, we end the proof of Theorem 3.3

Acknowledgment. This work was partially supported by the National Natural Science Foundation of China under grants 11501424 and 11701535.

\section{References}

[1] J. Apraiz, L. Escauriaza. Null-control and measurable sets. ESAIM: COCV, 19 (2013), 239254.

[2] J. Apraiz, L Escauriaza, G. Wang, C. Zhang. Observability inequalities and measurable sets. J. Eur. Math. Soc., 16 (2014), 2433-2475.

[3] V. Barbu. Exact null internal controllability for the heat equation on unbounded convex domains. ESAIM: COCV, 20 (2014), 222-235.

[4] C. Bardos, G. Lebeau, J. Rauch. Sharp sufficient conditions for the observation, control, and stabilization of waves from the boundary. SIAM J. Control and Optim., 30 (1992), 1024-1065.

[5] A. Bonami, B. Demange. A survey on uncertainty principles related to quadratic forms. Collect. Math., (2006), 1-36.

[6] N. Burq, R. Joly. Exponential decay for the damped wave equation in unbounded domains. Commun. Contemp. Math., 18 (2016), 1650012.

[7] V. R. Cabanillas, S. B. de Menezes, E. Zuazua. Null controllability in unbounded domains for the semilinear heat equation with nonlinearities involving gradient terms. J. Optimiz. Theory App., 110 (2001), 245-264.

[8] P. Cannarsa, P. Martinez, J. Vancostenoble. Null controllability of the heat equation in unbounded domains by a finite measure control region. ESAIM: COCV, 10 (2004): 381-408.

[9] T. Duyckaerts, L. Miller. Resolvent conditions for the control of parabolic equations. J. Funct. Anal., 263 (2012), 3641-3673.

[10] S. Ervedoza. Control and stabilization properties for a singular heat equation with an inversesquare potential. Commun. Partial Diff. Eq., 33 (2008), 1996-2019.

[11] L. Escauriaza, F. J. Fernández, S. Vessella. Doubling properties of caloric functions. Appl. Anal., 85 (2006), 205-223. 
[12] L. Escauriaza, C. Kenig, G. Ponce, L. Vega. On uniqueness properties of solutions of Schrödinger equations. Commun. Partial Diff. Eq., 31 (2006), 1811-1823.

[13] L. Escauriaza, C. Kenig, G. Ponce, L. Vega. Hardy's uncertainty principle, convexity and Schrödinger evolutions. J. Eur. Math. Soc. 10 (2008), 883-907.

[14] L. Escauriaza, C. Kenig, G. Ponce, L. Vega. Uniqueness properties of solutions to Schrödinger equations. Bull. Amer. Math. Soc. 49 (2012), 415-442.

[15] L. Escauriaza, C. E. Kenig, G. Ponce and L. Vega. Hardy Uncertainty Principle, Convexity and Parabolic Evolutions, Commun. Math. Phys. 346 (2016) 667-678.

[16] L. Escauriaza, S. Montaner, C. Zhang. Observation from measurable sets for parabolic analytic evolutions and applications. J. Math. Pures Appl, 104 (2015): 837-867.

[17] G. B. Folland, A. Sitaram. The uncertainty principle: a mathematical survey. J. Fourier Anal. Appl., 3 (1997), 207-238.

[18] A. V. Fursikov, O. Y. Imanuvilov. Controllability of evolution equations. Seoul National University, 1996.

[19] V. Havin, B. Jöricke. The Uncertainty Principle in Harmonic Analysis. Springer Science \& Business Media, 2012.

[20] A. Ionescu, C. Kenig. $L^{p}$ Carleman inequalities and uniqueness of solutions of nonlinear Schrödinger equations. Acta Math. 193 (2004), 193-239.

[21] A. Ionescu, C. Kenig. Uniqueness properties of solutions of Schrödinger equations. J. Funct. Anal., 232 (2006), 90-136.

[22] R. Joly, C. Laurent. Stabilization for the semilinear wave equation with geometric control condition. Analysis \& PDE, 6 (2013), 1089-1119.

[23] V. È. Kacnelśon. Equivalent norms in spaces of entire functions. Mathematics of the USSRSbornik, 21 (1973), 33.

[24] O. Kovrijkine. Some results related to the Logvinenko-Sereda theorem. Proc. Amer. Math. Soc., 129 (2001), 3037-3047.

[25] S. Huang, A. Soffer. Uncertainty principle, minimal escape velocities and observability inequalities for schrö dinger equations. arXiv:1709.09485, 2017.

[26] C. Laurent. Internal control of the Schrödinger equation. Math. Control Relat. F., 4 (2014), $161-186$.

[27] G. Lebeau, L. Robbiano. Contrôle exact de léquation de la chaleur. Commun. Partial Diff. Eq., 20 (1995), 335-356.

[28] G. Lebeau and E. Zuazua, Null-controllability of a system of linear thermoelasticity, Arch. Rational Mech. Anal., 141 (1998), pp. 297-329.

[29] J. Le Rousseau, G. Lebeau, P. Terpolilli, E. Trélat. Geometric control condition for the wave equation with a time-dependent observation domain. Analysis \& PDE, 10 (2017), 983-1015.

[30] J. Le Rousseau, I. Moyano. Null-controllability of the Kolmogorov equation in the whole phase space. J. Differ. Equ., 260 (2016), 3193-3233. 
[31] V. N. Logvinenko, J. F. Sereda. Equivalent norms in spaces of entire functions of exponential type. Teor. FunkciiFunkcional. Anal. i Prilozen. Vyp, 20 (1974), 102-111.

[32] A. Lopez, X. Zhang, and E. Zuazua. Null controllability of the heat equation as singular limit of the exact controllability of dissipative wave equations, J. Math. Pures Appl., 79 (2000), $741-808$.

[33] Q. Lü. A lower bound on local energy of partial sum of eigenfunctions for Laplace-Beltrami operators. ESAIM COCV, 19 (2013) 255-273.

[34] Q. Lü, Z. Yin. Unique continuation for stochastic heat equations. ESAIM: COCV, 21 (2015), 378-398.

[35] S. Micu, E. Zuazua. On the lack of null-controllability of the heat equation on the half-line. Trans. Amer. Math. Soc., 353 (2001), 1635-1659.

[36] S. Micu, E. Zuazua. On the lack of null-controllability of the heat equation on the half space. Port. Math., 58 (2001), 1-24.

[37] L. Miller. On the null-controllability of the heat equation in unbounded domains. B. Sci. math., 129 (2005), 175-185.

[38] L. Miller. Unique continuation estimates for the Laplacian and the heat equation on noncompact manifolds. Math. Res. Lett., 12 (2005), 37-47.

[39] L. Miller. Unique continuation estimates for sums of semiclassical eigenfunctions and nullcontrollability from cones, Preprint, 2009.

[40] F. L. Nazarov. Local estimates for exponential polynomials and their applications to inequalities of the uncertainty principle type, Algebra i Mathematicheskiy Analiz 5 (1993), 3-66.

[41] B.P. Paneyah. Some estimates of functions of exponential type and apriori estimates for gerneral dikerential operators, Uspekhi mat.nauk 21 N3 (1996) 75-114 (Russian).

[42] B.P. Paneyah. On some theorems of Paley-Wiener type. Doklady AN SSSR 138 N1 (1961) 47-50 (Russian).

[43] K. D. Phung, G. Wang. Quantitative unique continuation for the semilinear heat equation in a convex domain. J. Funct. Anal., 259 (2010), 1230-1247.

[44] K. D. Phung, G. Wang. An observability estimate for parabolic equations from a measurable set in time and its applications. J. Eur. Math. Soc, 15 (2013), 681-703.

[45] K. D. Phung, G. Wang, Y. Xu. Impulse output rapid stabilization for heat equations. J. Diff. Equ., 263 (2017), 5012-5041.

[46] K. D. Phung, L. Wang, C. Zhang. Bang-bang property for time optimal control of semilinear heat equation. Ann. Inst. H. Poincar'e Anal. Non Lineaire, 31 (2014), 477-499.

[47] J. Rauch, M. Taylor. Exponential decay of solutions to hyperbolic equations in bounded domains. Indiana Univ. Math. J. 24 (1974), 79-86.

[48] M. Reed and B. Simon. Methods of Modern Mathematical Physics I: Functional Analysis, Academic Press, 1980. 
[49] A. Reznikov. Sharp constants in the PaneyahCLogvinenkoCSereda theorem. Comptes Rendus Mathematique, 348 (2010), 141-144.

[50] B. Simon. Schrödinger semigroups. Bull. Amer. Math. Soc., 7 (1982), 447-526.

[51] S. Vessella. A continuous dependence result in the analytic continuation problem. Forum Math. 11, 6 (1999) 695-703.

[52] G. Wang, M. Wang, Y. Zhang. Observability and unique continuation inequalities for the Schrödinger equation. To appear in J. Eur. Math. Soc., arXiv preprint arXiv:1606.05861, 2016.

[53] G. Wang, C. Zhang. Observability inequalities from measurable sets for some abstract evolution equations. SIAM J. Control Optim., 55 (2017), 1862-1886.

[54] C. Zhang. Quantitative unique continuation for the heat equation with Coulomb potentials. arXiv preprint arXiv:1707.07744, 2017.

[55] Y. Zhang. Unique continuation estimates for the Kolmogorov equation in the whole space. Comptes Rendus Mathematique, 354 (2016), 389-393.

[56] E. Zuazua. Exponential decay for the semilinear wave equation with localized damping in unbounded domains. J. Math. Pures Appl, 70 (1991), 513-529. 